\title{
Inversion of calcite twin data for paleostress orientations and magnitudes: A new technique tested and calibrated on numerically-generated and natural data
}

\author{
Parlangeau Camille 1, 2, ${ }^{*}$, Lacombe Olivier ${ }^{1}$, Schueller Sylvie ${ }^{2}$, Daniel Jean-Marc ${ }^{2}$
}

${ }^{1}$ UPMC Univ Paris 06, Sorbonne Univ, CNRS, Inst Sci Terre Paris iSTeP, 4 PI Jussieu, F-75005 Paris, France.

2 IFP Energies Nouvelles, 1-4 Ave Bois Preau, F-92500 Rueil Malmaison, France.

* Corresponding author : Camille Parlangeau, email address : camille.parlangeau@gmail.com

\begin{abstract}
The inversion of calcite twin data is a powerful tool to reconstruct paleostresses sustained by carbonate rocks during their geological history. Following Etchecopar's (1984) pioneering work, this study presents a new technique for the inversion of calcite twin data that reconstructs the 5 parameters of the deviatoric stress tensors from both monophase and polyphase twin datasets. The uncertainties in the parameters of the stress tensors reconstructed by this new technique are evaluated on numerically-generated datasets. The technique not only reliably defines the 5 parameters of the deviatoric stress tensor, but also reliably separates very close superimposed stress tensors $\left(30^{\circ}\right.$ of difference in maximum principal stress orientation or switch between $\sigma 3$ and $\sigma 2$ axes). The technique is further shown to be robust to sampling bias and to slight variability in the critical resolved shear stress. Due to our still incomplete knowledge of the evolution of the critical resolved shear stress with grain size, our results show that it is recommended to analyze twin data subsets of homogeneous grain size to minimize possible errors, mainly those concerning differential stress values. The methodological uncertainty in principal stress orientations is about $\pm 10^{\circ}$; it is about \pm 0.1 for the stress ratio. For differential stresses, the uncertainty is lower than $\pm 30 \%$.
\end{abstract}

Applying the technique to vein samples within Mesozoic limestones from the Monte Nero anticline (northern Apennines, Italy) demonstrates its ability to reliably detect and separate tectonically significant paleostress orientations and magnitudes from naturally deformed polyphase samples, hence to fingerprint the regional paleostresses of interest in tectonic studies. 


\section{Highlights}

- We propose a new inversion method of calcite twin datasets. Inversion method is calibrated using numerically-generated datasets. The uncertainties and validity limits of this new method are defined. - Inversion of calcite twin datasets provides the 5 parameters of the deviatoric stress tensors. Natural case is used to test the validity of the method.

Keywords : Inversion method, Calcite twin, Paleostress 
Defining deformation mechanisms in the upper crust as well as their driving stresses are key

32 scientific and technical issues. Quantifying stresses allows to better understand the mechanical be-

33 havior of geological materials and to decipher tectonic mechanisms, from those related to plate mo-

34 tions at a large scale to those causing jointing and faulting or even microstructures at a smaller scale.

35 However, the way stress orientations and magnitudes actually evolve in naturally deforming rocks

36 over time scales of several tens of millions years is still a pending question, and even sophisticated

37 geomechanical models require stress constraints in order to be accurately calibrated.

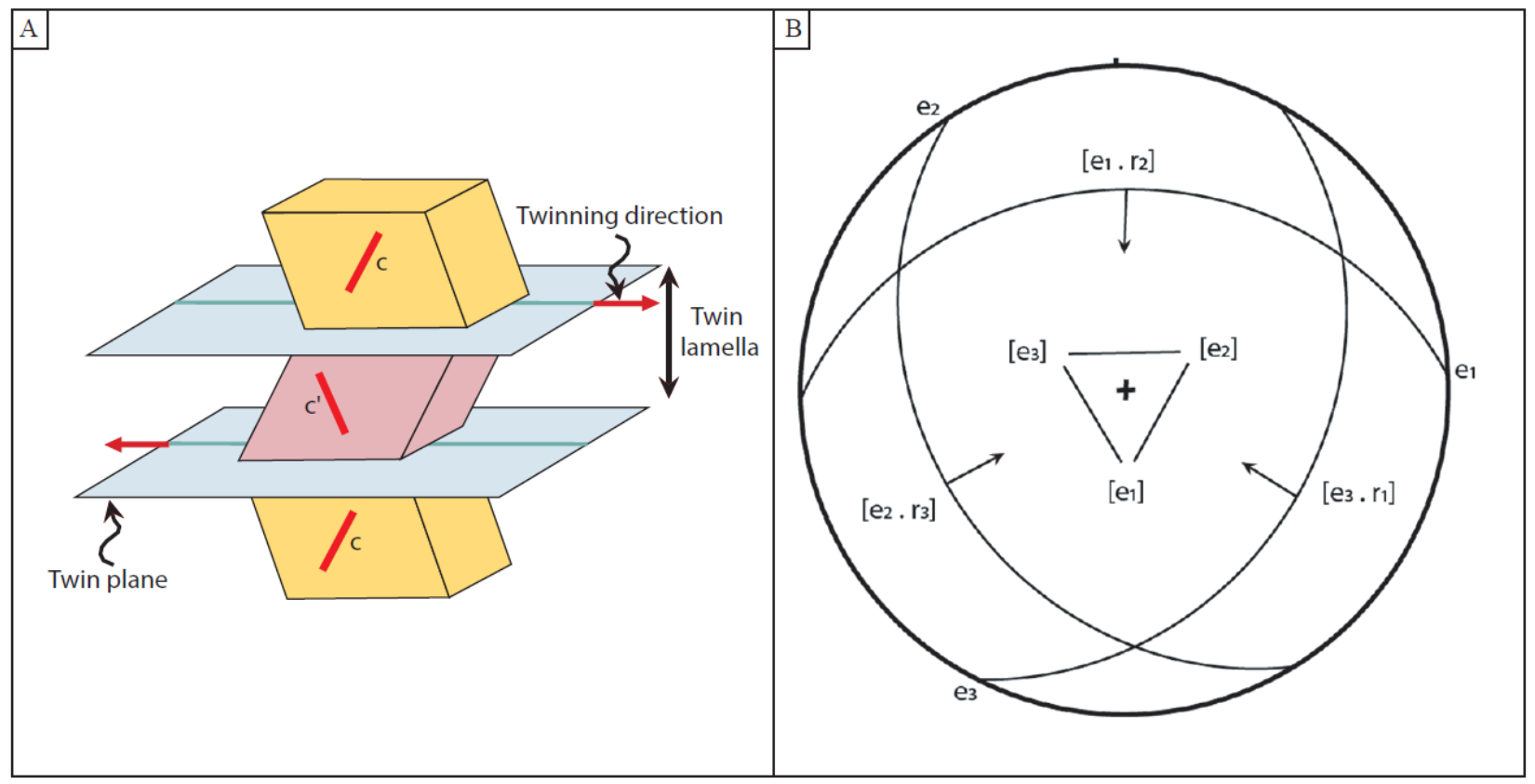

Figure 1: Scheme of a twin lamella (pink) in a calcite crystal (yellow). B) Stereographic projection (lower hemisphere, equal area) of the e-twinning system of the calcite crystal. The optical axis C is vertical at the center of the diagram. The poles of the three twin plane sets are [e 1$],\left[\mathrm{e}_{2}\right]$ and [ $\left.\mathrm{e}_{3}\right]$. The planes of twinning are the large circles; they contain the twinning direction $\left[\mathrm{e}_{\mathrm{i}}: \mathrm{r}_{\mathrm{j}}\right]$; for each twin plane, the arrow is parallel to the twinning direction ; its head indicates that the upper part of the crystal moves upward, toward the $\mathrm{C}$ axis, as a reverse microfault (After Turner et al., 1954). 
In order to decipher the tectonic evolution and to provide constraints on the past states of

stress, methods of paleostress reconstructions based on the mechanical interpretation of various structural or petrographic elements in natural rocks have been set out. Among these methods, those based on the microstructural study of twinning in minerals have proven to be efficient in reconstructing stress (orientations and/or magnitudes) sustained by rocks during their tectonic history. These methods make use of the property that twinning depends on the magnitude of the shear stress which has been applied to them (Tullis, 1980). E-twinning is a common mechanism of plastic deformation in calcite aggregates deformed at low pressure and low temperature. E-twinning occurs in the host crystal by an approximation to simple shear in a particular sense and direction along specific crystallographic e planes (Fig. 1). Since calcite is widely encountered in sedimentary basins and mountain belts and is among the most sensitive mineral for twinning, it can be seen as an important paleostress indicator for the upper crust (e.g., Lacombe, 2010). Inversion of calcite twins for paleostress is thus a well-established approach to reconstruct paleostress orientations and magnitudes of differential stresses (Amrouch et al., 2010; Arboit et al., 2015; Kulikowski and Amrouch, 2017; Lacombe, 2001; Lacombe et al., 2009, 1990; Lacombe and Laurent, 1992; Rocher et al., 2000, 1996) in nature and is a promising tool to estimate principal stress magnitudes when combined with fracture analysis and rock mechanics (Amrouch et al., 2011; Arboit et al., in press; Lacombe, 2007, 2001) or stylolite roughness paleopiezometry (Beaudoin et al., 2016). Among the available techniques, the Calcite Stress Inversion Technique, CSIT (Table 1) (Etchecopar, 1984) allows the determination of the five parameters of the deviatoric stress tensor, i.e., principal stress orientations and differential stress magnitudes, the latter being strongly dependent on the (still debated) existence of a Critical Resolved Shear Stress (CRSS) for twinning (Burkhard, 1993; De Bresser and Spiers, 1997; Newman, 1994; Rowe and Rutter, 1990; Tullis, 1980).

Despite a wealth of successful regional paleostress reconstructions in polyphase tectonic settings (see Lacombe, 2010, and references therein), the application by Gagala (2009) of the CSIT to numerically generated calcite aggregates has cast some doubt on the ability of the technique to 

stated and remain to be better defined.

\begin{tabular}{ll}
\hline CSIT & Calcite stress inversion technique \\
CRSS & Critical resolved shear stress \\
$\tau_{s}$ & Applied resolved shear stress \\
$\tau_{\mathrm{a}}$ & Critical resolved shear stress \\
$\sigma_{1}, \sigma_{2}, \sigma_{3}$ & The principal stress \\
$\Phi$ & The stress ratio \\
$\mathrm{F}$ & The penalization function \\
$\tau_{s}^{\text {min }}$ & The smallest resolved shear stress applied on the last twinned plane taken into \\
$\tau_{s}^{j}$ & account by the inversion method \\
$\mathrm{L}^{2}-$ norm & The resolved shear stress applied on the jth untwinned plane \\
$\Delta_{1-3}$ & Distance calculated between two tensor in MPa \\
\hline
\end{tabular}

Table 1: List of symbols used.

efficiently separate superimposed tensors with close principal stress orientations. Moreover, methodological uncertainties in stress parameters as determined by CSIT have never been properly

\section{Table 1: List of symbols used.}

The goal of this study is to present and test a new scheme of inversion, called CSIT-2, partly inspired by the CSIT. The newly developed technique and its ability to efficiently separate superimposed stress tensors are tested on numerically-generated and naturally deformed monophase (only one tensor applied) and polyphase (two tensors applied) twin datasets. The results demonstrate the reliability of such an approach to derive the stress parameters of interest, hence to provide an efficient toolbox for tectonic studies.

\section{A brief review of existing methods to derive stress from calcite twins}

Since the pioneering work of Turner (1953), several methods of stress reconstruction have been developed on the basis of the analysis of calcite twin data (Etchecopar et al., 1981; Jamison and Spang, 1976; Laurent et al., 1990, 1981; Nemcok et al., 1999; Pfiffner and Burkhard, 1987; Yamaji, 2015a, 2015b).

The basis of the widely used paleopiezometric method of Jamison and Spang (1976) is that, in a sample without any preferred crystallographic orientation, the relative percentages of grains twinned on $0,1,2$ or 3 twin plane(s) depend on the applied differential stress value $\left(\sigma_{1}-\sigma_{3}\right)$. Since 
81 this relationship has been experimentally calibrated, knowing these relative percentages in a sample and under the hypothesis of a constant CRSS for twinning, the order of magnitude of $\left(\sigma_{1}-\sigma_{3}\right)$ can be estimated. The severe limitations of this method are that (1) it does not take into account the grain size dependence of twinning, (2) it applies only to uniaxial stresses, (3) it does not check before calculation whether twin data are related to one or more superimposed stress tensors and (4) it does not make it possible to relate differential stress estimates to a given stress regime since principal stress orientations are not determined (Lacombe, 2010).

Rowe and Rutter (1990) determined empiric laws between twinning incidence, twin volume fraction, twin density and experimentally applied differential stress to derive a calcite twinning paleopiezometer. The two first parameters are dependent on the grain size. The twin density is more easily used to calculate the differential stress because it does not depend on grain size. However, the law has been calibrated for temperature above $400^{\circ} \mathrm{C}$ and clearly overestimates the differential stress values when applied to samples deformed at low temperature (Ferrill,1998). In addition, this method shares the same limitations as the Jamison and Spang (1976) technique, which consists in not checking whether twin data are related to one or more superimposed stress tensors and not making possible to relate the differential stress estimates to a given state of stress.

Laurent et al.'s technique (1981) was the first to take into account both twinned and untwinned planes to find 5 parameters of the stress tensor. This technique uses the deviatoric stress tensor (based on the nearly null dependence of twinning on isotropic stress) and relies upon a Boolean calculation to determine the solution tensor. This method was seldom used because it is time consuming ( Laurent et al., 1990). Laurent et al. (1990) also proposed a new method which determines the 5 parameters of the deviatoric stress tensor. This technique uses a non-linear equation to determine the deviatoric stress tensor and is faster than Laurent et al. (1981) technique. These methods have not given rise to numerous applications in the literature.

The CSIT ( Etchecopar, 1984) method is to date the most used technique to retrieve the past 
stress tensors (e.g., Amrouch et al. 2010; Beaudoin et al. 2012, 2016; Lacombe and Laurent 1992; Lacombe 2001, 2007; Lacombe et al. 2009; Rocher et al., 1996, 2000). The inversion process is very similar to the one used for fault slip data (Etchecopar, 1984), since twin gliding along the twinning direction within the twin plane is geometrically comparable to slip along a slickenside lineation within a fault plane. The basic assumptions are: (1) the existence of a Critical Resolved Shear Stress (CRSS) for twinning and (2) a potential twin plane is twinned (respectively, untwinned) if the resolved shear stress applied on it is greater (respectively, lower) than the CRSS. The inversion process takes into account both the twinned and untwinned planes and provides the 5 parameters of the deviatoric stress tensor, i.e., principal stress orientations and differential stress magnitudes. It should be noted that the value of the CRSS depends on grain size as well as on internal grain deformation since calcite hardens once twinned (Newman, 1994; Tullis, 1980; Turner et al. 1954). However the evolution of CRSS with grain size has not been well constrained yet. The CSIT technique has been successfully tested on experimentally deformed natural samples (Lacombe and Laurent, 1996; Laurent et al., 2000) and the results have shown maximum deviations of computed principal stress orientations compared to experimentally applied ones of $5-7^{\circ}$ for monophase and $7-11^{\circ}$ for polyphase cases. In 2009 , Gągała questioned the ability of this technique to reliably reconstruct the orientations of the principal stress axes and the stress ratio of close superimposed stress tensors. The penalization function used to refine the tensor solution is considered to be too restrictive by Rez and Melichar (2010) and Yamaji (2015b), especially when dealing with natural samples where optical measurement bias may occur. For Rez and Melichar (2010), the penalization function of CSIT (see section 3.1) is strongly dependent on the compatible twinned and the incompatible untwinned planes with the stress tensor solution. In addition, the space of solutions with a penalization function of 0 is too large. So, they proposed a new penalization function with sharper maxima, depending on the number of compatible twinned planes, the number of compatible untwinned planes and the number of incompatible untwinned planes. Note however that the CSIT with this refined penalization function has never been applied to polyphase twin datasets. 
Nemcok et al. (1999) technique works for both calcite twin and fault-slip data. The approach

consists in searching all 3D tensors which can activate or re-activate a twin plane. They analyze clusters of twin data to group them in sets based on their response to one or multiple stress tensors. Each subset is tested to establish monophase solution twin datasets. Polyphase sets are divided into monophase subsets. Each subset is analyzed to obtain the reduced stress tensor (orientation of principal stress axes and stress ratio). The study of Gagała (2009) demonstrates that this method is however not suitable for treating polyphase data.

The most recent stress inversion technique to date has been proposed by Yamaji (2015b). This technique, based on the generalized Hough transform, provides like CSIT, the 5 parameters of the deviatoric stress tensor. The preliminary exploration of the extent to which calcite twinning may constrain stress (Yamaji, 2015a) demonstrates that twinned planes better constrain the stress tensor than untwinned planes and that differential stress estimates are poorly resolved for differential stresses greater than 50-100 $\mathrm{MPa}$. This technique seems to separate superimposed stress tensors only if the intersection between their spherical caps is nonexistent or small. Spherical caps correspond to the data points on a unit sphere based on the five-dimensional stress space; the deviatoric stress tensors responsible for twinning are thus denoted by the size and position of the spherical cap (see Yamaji, 2015a for details). Comparing his new technique to existing ones, Yamaji (2015a) states that the CSIT appears to be unstable, mainly due to the selection method of the solution tensors, which is only dependent on the last twinned plane taken into account in each solution tensor tested (see the theory of CSIT below). The limitation of Yamaji's technique is that it has not to date been tested on experimentally or naturally deformed samples.

An outcome of this short review is that the analysis of calcite twin data makes it possible to reliably obtain the 5 parameters of the deviatoric stress tensor under specific assumptions in monophase twin datasets. However, the challenge of all techniques is to reliably separate and reconstruct superimposed stress tensors as from polyphase datasets. There is especially a consensus toward the difficulty to detect and separate tensors which are very close in terms of principal stress 
158

159

160

161

162

163

164

165

166

167

168

169

170

171

172

173

174

175

176

177

178

179

180

orientations and differential stress magnitudes. These questions are addressed hereinafter through the setup and calibration of a new inversion scheme, CSIT-2. Its application to synthetic (numerically generated) calcite twin data allows to define the methodological uncertainties and the applicability domains of the new technique as well as the influence of heterogeneities commonly found in natural samples. The technique is finally applied to a naturally deformed polyphase sample to check its ability to reconstruct the regional paleostresses.

\section{A new calcite twin inversion scheme (CSIT-2): methodology}

\subsection{Theory and basic equations of CSIT (Etchecopar, 1984)}

As said above, the principle of CSIT is to invert a calcite twin dataset for stress. The basic underlying hypothesis is that a potential e-twin plane is twinned if and only if the resolved shear stress applied on the direction of the twinning exceeds the critical resolved shear stress value $\tau_{a}(\mathrm{CRSS})$ :

- If $\tau_{s} \geq \tau_{a}$, then the plane is twinned. (1)

- If $\tau_{s}<\tau_{a}$, then the plane remains untwinned. (2)

with $\tau_{s}$, the resolved shear stress applied along the gliding direction of the e-plane. The optimally oriented twin plane will be activated if the applied differential stress is equal to, or greater than, $2 \tau_{a}$.

The basic assumptions are that strain and stress are coaxial (low strain conditions), the stress field is homogeneous at the grain scale and twinning is a non-reversible process.

The principle of the inversion of calcite twin dataset is to find a stress tensor (or several stress tensors) which verifies these two inequalities for the largest number of twinned planes and the whole set of untwinned planes. The solution has the form of a reduced stress tensor with 4 parameters: the orientations of principal stress axes $\left(\sigma_{1}, \sigma_{2}, \sigma_{3}\right)$ and the stress ratio $(\Phi)$ :

$1 \geq \Phi=\frac{\sigma_{2}-\sigma_{3}}{\sigma_{1}-\sigma_{3}} \geq 0(3)$

The differential stress $\left(\sigma_{1}-\sigma_{3}\right)$ is normalized to 1 , so the normalized resolved shear stress applied 
on each twin plane varies within $[-0.5 ; 0.5]$.

The inverse problem consists in finding the stress tensor that best explains the spatial distribution of measured twinned and untwinned planes. The first step consists in an arbitrary choice of a percentage of twinned planes to be explained. The resolved shear stresses are calculated on the twinned and untwinned planes, which are ranked as a function of the decreasing resolved shear stress. In theory, the solution tensor should meet the requirement that all the twinned and untwinned planes should be consistent with it. Thus, all twinned planes should sustain a resolved shear stress $\left(\tau_{\mathrm{s}}\right)$ larger than that exerted on all the untwinned planes. The sorting allows to determine rapidly whether some untwinned planes are incompatible with the tensor (i.e., the resulting resolved shear stress is greater than that for some compatible twinned planes).

The second step of the process therefore consists in calculating a penalization function, $f$, ideally equal to 0 , which is defined as:

$$
f=\sum_{j=1}^{j=n}\left(\tau_{s}^{j}-\tau_{s}^{\min }\right)
$$

where $\tau_{s}^{\min }$ is the smallest resolved shear stress applied on the twinned planes compatible with the tensor, and $\tau_{s}^{j}$ is the resolved shear stresses applied on the $\mathrm{j}$ untwinned planes such that $\tau_{s}^{j}>\tau_{s}^{\min }$. The penalization function increases if incompatible untwinned planes are incorporated in the solution. The optimal tensor is obtained when the maximum number of twinned planes and the minimum number of incompatible untwinned planes are incorporated in the solution.

This process therefore yields the orientation of the 3 principal stress axes, the stress ratio and a nondimensional differential stress. This non-dimensional differential stress, $\frac{\left(\sigma_{1}-\sigma_{3}\right)}{\tau_{a}}$, is such that,

$$
\frac{\left(\sigma_{1}-\sigma_{3}\right)}{\tau_{a}}=\frac{\left(\sigma_{1}-\sigma_{3}\right)_{\text {normalized }}}{\tau_{s}^{\min }}=\frac{1}{\tau_{s}^{\min }}
$$

Under the assumption of a known and constant CRSS, $\tau_{a}$, the actual differential stress is 
given by equation 5 :

$$
\left(\sigma_{1}-\sigma_{3}\right)=\frac{\tau_{a}}{\tau_{s}^{\min }}
$$

\subsection{New inversion scheme (CSIT-2): motivation and principle}

If the basic principle of CSIT-2 remains more or less the same as the CSIT for the calculation

207 of the penalization function, the major difference lies in the automatic detection of one or several

208 tensors. The choice has been made to systematically cover the space with a regular interval of $10^{\circ}$ for

209 the 3 Euler's angles (defining the orientation of the stress tensor axes) and a fixed stress ratio value

210 of 0.5 . The total amount of tested tensors is of 5832 tensors in CSIT-2.

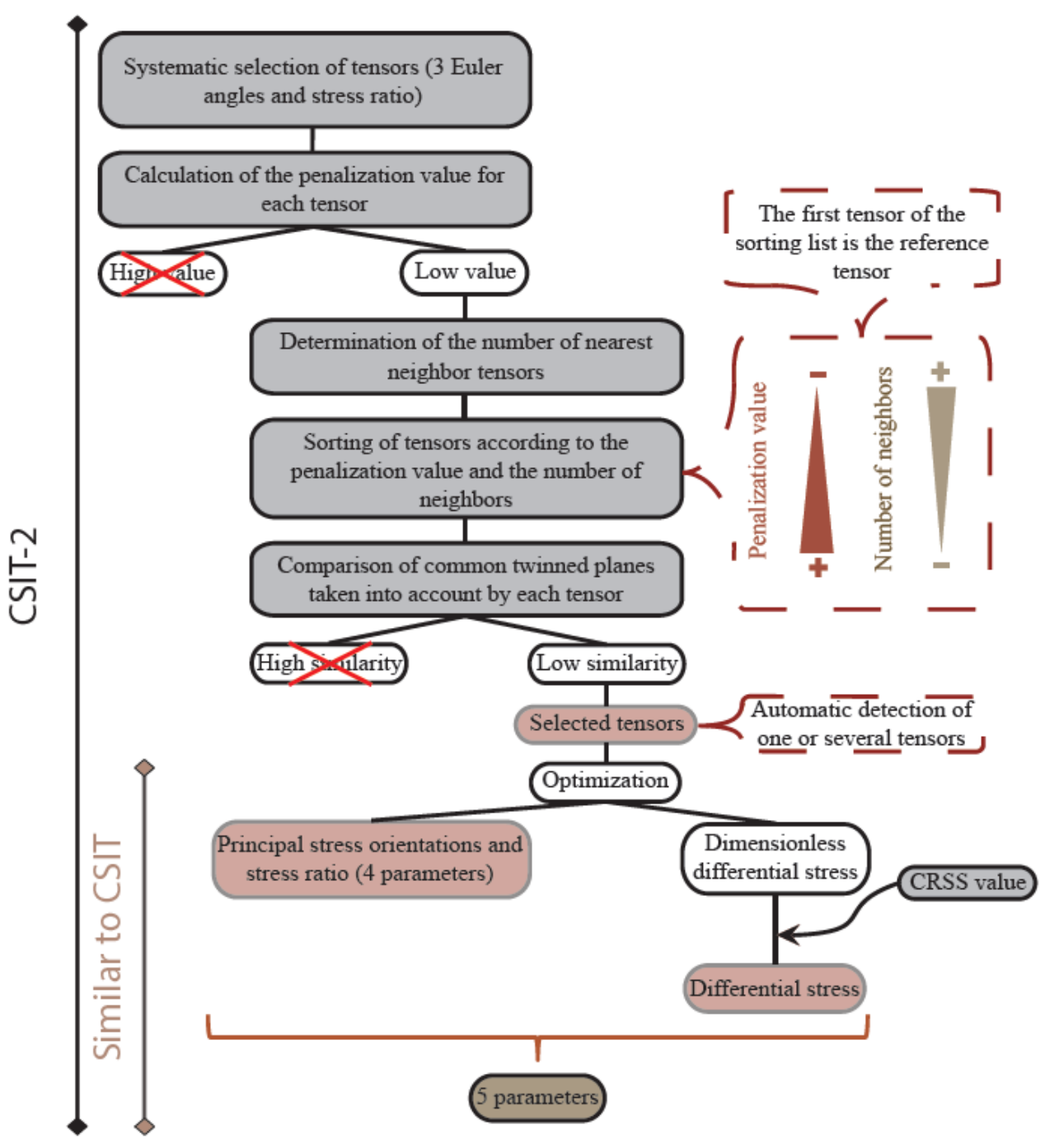

Figure 2: Workflow of CSIT-2 for inversion of calcite twin datasets.

211 The tensor that is retained by the CSIT method for further optimization is the tensor presenting 
212 the minimum value of the penalization function. This means that if several tensors with the same

213 minimum penalization function exist, only one will be arbitrarily chosen for the inversion. However,

214 in case of several tensors with the same low penalization function (ideally 0 , corresponding to a 215 solution with incorporation of no incompatible untwinned planes), it is interesting to keep these 216 different tensors, which might correspond to superimposed stress tensors. Contrary to CSIT, the 217 CSIT-2 allows to automatically detect the presence of one or several tensors. For that purpose, the 218 following steps are performed (Fig. 2):

(1) The user defines a starting percentage of twinned planes to be explained $(20 \%$ recommended).

(2) The program will test systematically different tensors with the 3 Euler's angles being sampled every $10^{\circ}$ and a stress ratio of 0.5 .

(3) The penalization function is calculated for each stress tensor (equation 4).

(4) The stress tensors with a penalization function larger than a limit fixed by the user are discarded.

(5) Each tensor is weighted by the number of its nearest neighbors, based on the calculation of the angular distance (Yamaji and Sato, 2006). This step is important to automatically detect the different clusters of tensors with low penalization function values.

(6) The tensors are sorted in descending order based on the number of nearest neighbors and then in ascending order based on penalization function.

(7) The "best" tensors are selected: the first tensor of the list is the reference one. The reference tensor has the largest number of neighbors and the lowest penalization function value. The other tensors are kept if the percentage of shared twinned planes does not exceed a percentage threshold chosen by the user.

(8) The selected tensors are then optimized for different percentages of twinned planes to be 
explained using the Rosenbrock (1960) optimization method.

Thus, the choice of the retained tensors is based on different criteria: percentage of twinned 238 planes to be explained (step 1), penalization function (steps 4 and 6), angular distance (step 5) and 239 similarity criterion (step 7). The starting percentage of twinned planes to be explained is generally $24020 \%$. This value has been chosen because for a lower percentage, optical measurement uncertainties 241 and grain-scale heterogeneities as expected in naturally deformed samples could generate too much 242 noise and thus too many stress tensors could satisfactorily match the solution. If a percentage higher 243 than $20 \%$ is chosen there is a risk to miss potentially superimposed tensors by only detecting the best 244 expressed tensor. This percentage of twinned planes to explain has been chosen after several 245 numerical tests to configure the different parameters depending on the user (the starting percentage 246 of twinned planes to explain, the angular distance and the percentage of shared twinned planes 247 between two tensors to determine if they are similar or not). Finally, in case of superimposed stress 248 tensors with a high degree of similarity, it is possible that the inversion process may select an average 249 tensor (i.e., resulting from averaging the two applied tensors) in addition to the two applied tensors. 250 However, this average tensor is easy to identify and to discard because the technique also yields the 251 two applied tensors.

In order to automatically determine the different "best" tensors, it is necessary to highlight the 253 clusters of tensors with low penalization function values. For that purpose, several steps are needed. 254 The angular distance is calculated between all the selected tensors using the angular stress distance defined by Yamaji and Sato (2006). Each tensor is weighed by its penalization function as well as by the number of tensors within an angular distance of less than $30^{\circ}$ (Yamaji and Sato, 2006). This $30^{\circ}$ value has been retained because it involves a low variation of orientation of the principal stress axes and a possible variation of the stress ratio of less than 0.5 . This step is of first importance and makes 259 it possible to determine clusters with the highest density of tensors and a low penalization function 260 value. 
The first tensor on the sorted list is used as the reference tensor. It explains at least the chosen

$20 \%$ of twinned planes with possible incorporation of incompatible untwinned planes. The set of twinned planes explained by other tensors are compared to the set explained by this reference tensor. The parameter of similarity is defined as the number of common twinned planes between the first and the second tensor divided by the total number of twinned planes explained by the second tensor. A new tensor is retained if it involves less than $70 \%$ of similarities (for detailed explanations, see subsection 3.6). This makes it possible to detect very close tensors that consequently share a high percentage of twinned planes. The twinned planes explained by this second tensor are recorded and each other tensor is compared in the same way to determine a possible third tensor, and so on.

Following this similarity criterion, few tensors are selected. Then they are further tested against the twin dataset by increasing the percentage of twinned planes to be explained. For each percentage, an optimization is carried out (Rosenbrock, 1960). The ultimate goal is to explain the largest number of twinned planes and untwinned planes with an as low as possible penalization function value. The solution tensors are retained on the basis of the trend of evolution of their parameters: orientations of principal stress axes, penalization function, $\tau_{s}^{\min }$ value, stress ratio and number of incompatible untwinned planes against the increasing number of twinned planes to be explained. The first criterion considered is the stability of the principal stress axis orientations. If they are stable despite the increasing number of twinned planes to be explained, the second criterion is the stability of the penalization function together with the evolution of the percentage of incorporated incompatible untwinned planes. This last parameter is defined as being the number of incompatible untwinned planes divided by the number of twin planes taken into account by the tensor (compatible twinned planes + incompatible untwinned planes). The solution then corresponds to the percentage of twinned planes to be explained that is marked by the break in slope of the penalization function value. If this percentage corresponds to the break in slope of the incorporation of incompatible untwinned planes, then the stability of the stress ratio and of the resolved shear stress value should be taken into account to ultimately refine the solution. 


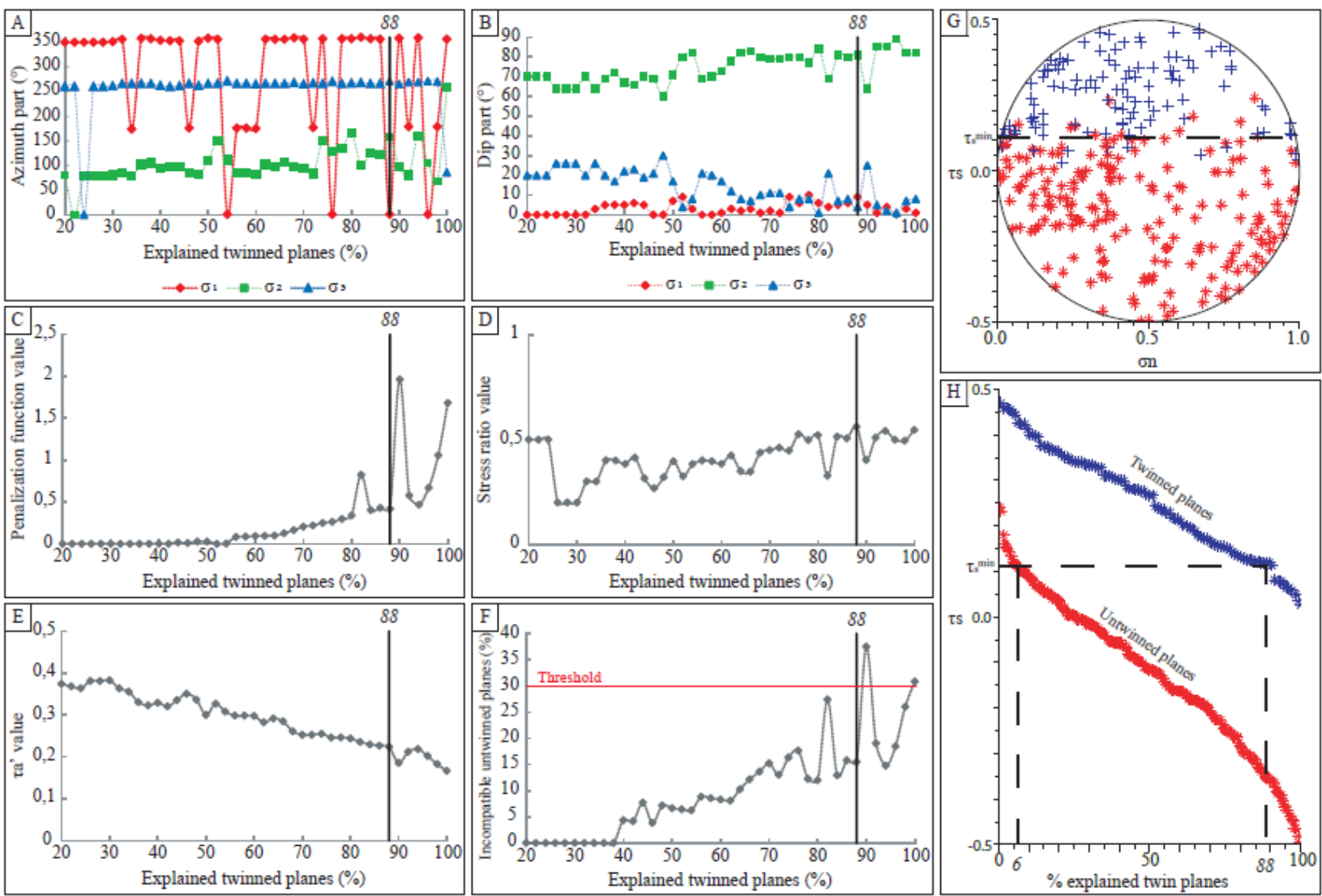

Figure 3: Graphics showing the variation of the penalization function value with the stress ratio for a same orientation stress axis ( $\sigma_{1}: \mathrm{N} 180-0$ and $\sigma_{3}$ : N270-0) and an applied stress ratio of 0.3 that has been registered after the added loop (see details in the first paragraph part 3.2) for A) 20\%, B) $70 \%$ and C) $80 \%$ of explained twinned planes.

An example in Figure 3 illustrates how the best solution (maximum number of twinned planes and minimum number of incompatible untwinned planes) is defined. Increasing the percentage of twinned planes to be explained does not affect here the principal stress orientations which remain very stable (Fig. 3A \& B), but it affects the $\tau_{s}^{\min }$ value (Fig. 3E) and thus the differential stress value (equation 5). Figure 3C represents the evolution of the penalization function with the increasing percentage of twinned planes to be explained. This curve is very similar to the curve describing incorporation of incompatible untwinned planes (Fig. 3F). In this example the best choice is the solution at $88 \%$ of explained twinned planes because the slope of the penalization function curve greatly increases above this percentage. It means that the incorporation of incompatible untwinned planes becomes too high beyond this percentage. Figures $3 \mathrm{D}$ and $3 \mathrm{E}$ display the evolution of the stress ratio value and of the $\tau_{s}^{\min }$ value used to calculate the differential stress (equation 5). For the 
stress ratio curve the solutions between $20 \%$ and $36 \%$ of explained twinned planes are not stable.

Above $36 \%$ of explained twinned planes, the stress ratio is stabilized at about 0.5 . Figure $3 \mathrm{G}$ represents the pseudo-Mohr circle showing $\tau_{\mathrm{s}}$ against $\sigma_{\mathrm{n}}$ and figure $3 \mathrm{H}$ displays the evolution of $\tau_{\mathrm{s}}$ for untwinned and twinned planes as a function of the percentage of twinned planes to be explained (calculated based on the total amount of untwinned planes). Both figures provide visualization of $\tau_{s}^{\min }$. As shown, the value of $\tau_{s}^{\text {min }}$ corresponds to a slight incorporation of untwinned planes in the solution $(<10 \%$, Fig. $3 \mathrm{H})$. In addition, these incompatible untwinned planes are plotted in figure $3 \mathrm{G}$ close to line representing the retained $\tau_{s}^{\min }$; this means that these incompatible untwinned planes

The solution is thus defined with confidence.
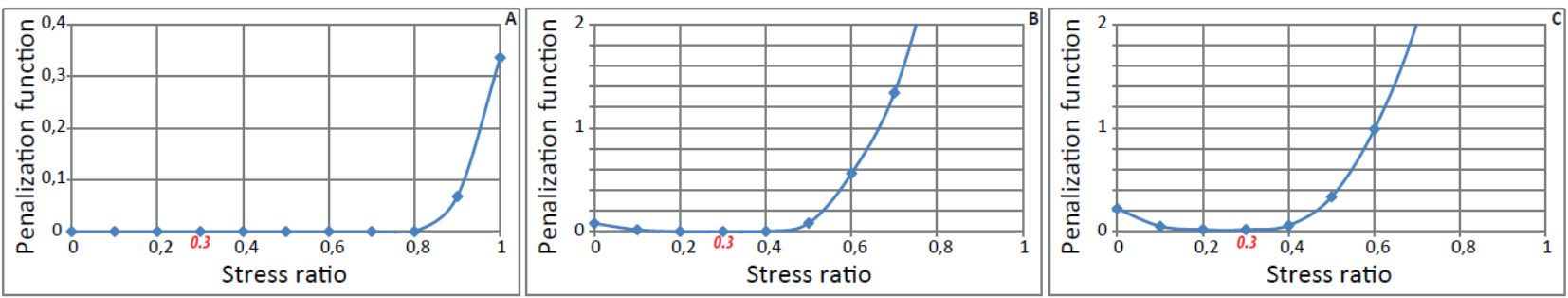

Figure 4: Determination of the best solution tensors of CSIT-based inversion on the basis of the evolution of parameters used during inversion process. The evolution of the parameters is shown as a function of the $\%$ of twinned planes to be explained by the tensor solution. The applied tensor is a N-S strike-slip regime $\left(\sigma_{1}=\mathrm{N} 180-0 ; \sigma_{3}=\mathrm{N} 270-0\right)$ with a stress ratio of 0.5 and a differential stress of $35 \mathrm{MPa}$ (test M9 in Table 1).

During the initial detection step, the choice to fix the stress ratio at 0.5 (following in that Yamaji, 2015b) was made (1) to reduce the computation time, and (2) because the inversion tends in polyphase cases to be stuck at tensors with extreme stress ratio ( 0 or 1$)$ that account for a larger number of twin data. However, to make sure this choice does not bias the determination of stress ratio,

312 a loop has been added before the optimization (step 8) which increases the stress ratio value by step 313 of 0.1 from 0 to 1 . The first test is a monophase case with $\sigma_{1}=\mathrm{N} 180-0 ; \sigma_{3}=\mathrm{N} 270-0$, a stress ratio of 3140.3 , a differential stress of $50 \mathrm{MPa}$ (Fig. 4). It can be observed that the inversion is not very sensitive 315 to the stress ratio at low percentage of twinned planes to explain. This suggests that at $20 \%$ of explained twinned planes (step 1), considering a fixed stress ratio (0.5) will not lead to artificially 
317 focus tensor solutions toward tensors with stress ratio of 0.5 hence to discard (step 4) those with stress

318 ratios different from this value .

With this initial detection step, the determination of stress tensors using CSIT-2 requires only a short computation time, about 15-20 minutes.

\subsection{Creation of synthetic calcite twin dataset}

\begin{tabular}{|c|c|c|c|c|c|c|c|c|c|c|c|c|c|c|c|c|}
\hline & M1 & M2 & M3 & M4 & M5 & M6 & M7 & M8 & M9 & M10 & M11 & M12 & M13 & M14 & M15 & M16 \\
\hline $\begin{array}{l}\text { Equivalent grain size } \\
\qquad(\mathrm{MPa})\end{array}$ & \multicolumn{6}{|c|}{10} & \multicolumn{2}{|c|}{$\begin{array}{c}(2 / 3 \\
\text { grains) } \\
5 \text { and } \\
(1 / 3 \\
\text { grains) } \\
15\end{array}$} & \multicolumn{3}{|c|}{$\begin{array}{c}\text { Gaussian } \\
\text { distribution } \\
\text { centered on } 10\end{array}$} & \multicolumn{3}{|c|}{$\begin{array}{c}\text { Gaussian } \\
\text { distribution } \\
\text { centered on } 8 \\
\text { and } 12\end{array}$} & \multicolumn{2}{|c|}{$\begin{array}{c}\text { Gaussian } \\
\text { distribution } \\
\text { centered on } \\
10\end{array}$} \\
\hline $\begin{array}{l}\text { Applied stress } \\
\text { orientation }\left({ }^{\circ}\right)\end{array}$ & \multicolumn{16}{|c|}{$\sigma_{1}: \mathrm{N} 180-0 ; \sigma 2: \mathrm{N} 90-90 ; \sigma 3: \mathrm{N} 270-0$} \\
\hline$\Phi$ & 0 & 0 & 0,5 & 0,5 & 1 & 1 & \multicolumn{10}{|c|}{0,5} \\
\hline$\Delta_{1-3}(\mathrm{MPa})$ & 25 & 75 & 25 & 75 & 25 & 75 & 50 & 75 & 35 & 50 & 75 & 35 & 50 & 75 & \multicolumn{2}{|c|}{50} \\
\hline Bias (\%) & & & & & & & & 0 & & & & & & & 10 & 25 \\
\hline
\end{tabular}

Table 2: Configuration of data tested for monophase cases.

The present method is first tested with numerically generated calcite twin datasets (Table 2 evaluate the performance, applicability domain and limitation of the new technique. Indeed, natural data have expectedly issues (spatial distribution of grains with different sizes, heterogeneity of stress within the aggregates due for instance to stress concentration at grain boundaries, inheritance - growth twins or earlier deformation twins - due to regional tectonic history), so that it is only after the synthetic experiments have been completed that the applicability of the CSIT- 2 technique to natural data will be evaluated.

For the simplest case (monophase synthetic sample with perfectly homogeneous grain size, table 2), one tensor is applied on randomly generated twin planes within grains with random 332 orientations of optical $\mathrm{C}$ axes (Fig. 5A). Each grain is assigned a CRSS value roughly simulating its 333 virtual size since twinning is grain size dependent (i.e, twinning is easier in large grains than in small 
grains). A 5-parameter (deviatoric) stress tensor is applied on the generated twin data, resulting in some e-twin planes being activated (twinned) depending on their orientation with respect to the applied stress (Fig. 5B).

\begin{tabular}{|c|c|c|c|c|c|c|c|c|c|c|c|c|c|c|c|c|c|c|c|}
\hline & B1 & B2 & B3 & B4 & B5 & B6 & B7 & B8 & \begin{tabular}{|l|l|l|} 
B9 & B10 & B11 \\
\end{tabular} & \begin{tabular}{|l|l|l|} 
B12 & B13 & B14 \\
\end{tabular} & 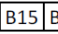 & \begin{tabular}{l|l|l|l|l} 
B16 & B17 \\
\end{tabular} & \begin{tabular}{|l|l|l|} 
B18 & B19 \\
\end{tabular} & B20 & \begin{tabular}{|l|l|l|} 
B21 & B22 & B23 \\
\end{tabular} & \begin{tabular}{|l|l|l|l|} 
B24 & B25 & B26 \\
\end{tabular} & B27 & B28 & B29 \\
\hline $\begin{array}{l}\text { Equivalent grain } \\
\text { size (MPa) }\end{array}$ & \multicolumn{19}{|c|}{ Gaussian distribution centered on 10} \\
\hline $\begin{array}{l}\text { Applied stress } \\
\text { orientation }\left({ }^{\circ}\right)\end{array}$ & \multicolumn{2}{|c|}{$\begin{array}{l}\sigma_{1}: \mathrm{N} 180-0 \\
\sigma 2: \mathrm{N} 90-90 \\
\sigma 3: \mathrm{N} 270-0 \\
\end{array}$} & \multicolumn{2}{|c|}{$\begin{array}{c}\sigma_{1}: N 30-0 \\
\sigma 2: N 120-90 \\
\sigma 3: N 300-0\end{array}$} & \multicolumn{2}{|c|}{$\begin{array}{l}\sigma_{1}: \text { N } 180-0 \\
\sigma 2: \text { N90-90 } \\
\sigma 3: \text { N270-0 }\end{array}$} & \multicolumn{2}{|c|}{$\begin{array}{c}\sigma_{1}: N 30-0 \\
\sigma 2: N 120-90 \\
\sigma 3: N 300-0\end{array}$} & $\begin{array}{c}\sigma_{1}: N 90-0 \\
\sigma 2: N 180-90 \\
\sigma 3: N 360-0 \\
\sigma\end{array}$ & $\begin{array}{c}\sigma_{1}: \mathrm{N} 180-0 \\
\sigma 2: \mathrm{N} 90-0 \\
\sigma 3: \mathrm{N} 270-90\end{array}$ & \multicolumn{4}{|c|}{$\begin{array}{c}\sigma_{1}: \text { N180-0 } \sigma 2: \text { N90-90 б3: } \\
\text { N270-0 }\end{array}$} & \begin{tabular}{|c|}
$\sigma_{1}: N 30-0$ \\
$\sigma 2: N 120-90$ \\
$\sigma 3: N 300-0$
\end{tabular} & $\begin{array}{l}\sigma_{1}: N 180-0 \\
\sigma 2: N 90-90 \\
\sigma 3: N 270-0\end{array}$ & \multicolumn{2}{|c|}{$\begin{array}{c}\sigma 1: \text { N30-0 } \\
\sigma 2: \text { N120-90 } \\
\sigma 3: \text { N300-0 }\end{array}$} & $\begin{aligned} \sigma_{1} & : N 180-0 \\
\sigma 2 & : N 90-0 \\
\sigma 3: & N 270-90\end{aligned}$ \\
\hline$\Phi$ & \multicolumn{10}{|c|}{0,5} & & 0 & 0,3 & & 0,7 & \multicolumn{4}{|c|}{0,5} \\
\hline$\Delta_{1-3}(\mathrm{MPa})$ & & & 50 & & 35 & 75 & 35 & 75 & \begin{tabular}{|l|l|l|}
35 & 50 & 75 \\
\end{tabular} & \begin{tabular}{|l|l|l|}
35 & 50 & 75 \\
\end{tabular} & 35 & \begin{tabular}{l|l|}
50 & 75 \\
\end{tabular} & \begin{tabular}{l|l|l}
35 & 50 & \\
\end{tabular} & 75 & \begin{tabular}{l|l|l}
35 & 50 & 1 \\
\end{tabular} & \begin{tabular}{l|r|}
35 & 50 \\
\end{tabular} & 50 & 75 & 35 \\
\hline
\end{tabular}

Table 3: Configuration of data tested for polyphase cases.

For the case of a polyphase synthetic sample (table 3) with homogeneous grain size, and in order to account for strain hardening, a second tensor is applied with each previously twinned grain being now assigned a CRSS increased by a value of $2 \mathrm{MPa}$ as proposed by Gągała (2009).

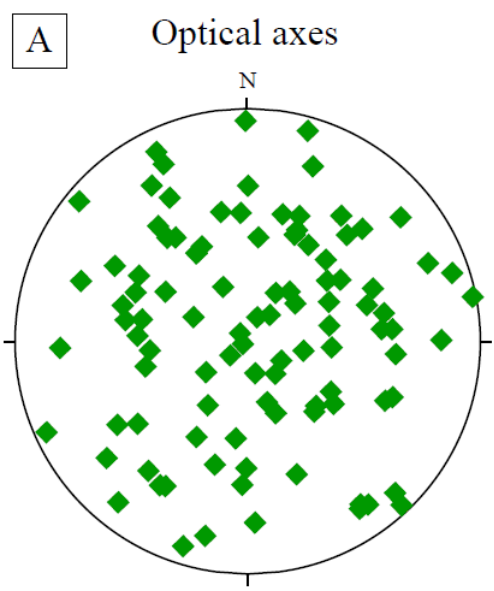

B Twin plane poles

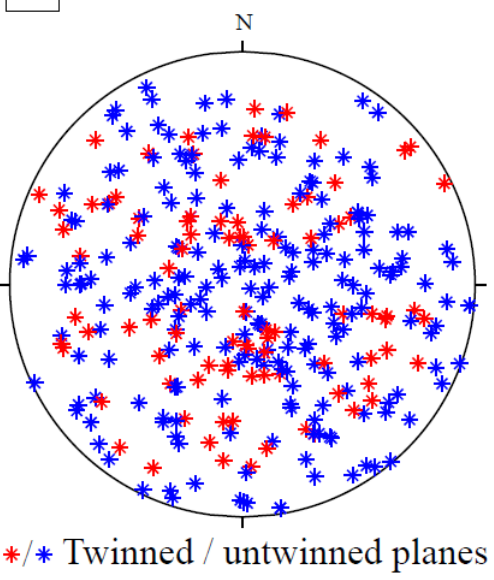

Figure 5: Wulff lower hemisphere stereographic projection of the random distribution $\mathrm{A}$ ) $\mathrm{C}$ axes and B) poles of twin planes in numerically-generated datasets. The applied stress tensor corresponds to: $\mathrm{N}-\mathrm{S} \sigma_{1}$ axis, E-W $\sigma_{3}$ axis, a stress ratio equal to 1 and differential stress equal to $75 \mathrm{MPa}$.

In order to simulate a heterogeneous grain size in both synthetic monophase and polyphase calcite aggregates, the choice was made to consider two virtual classes of grains with different sizes, simulated as two classes of grains with different assigned CRSS for twinning.

Finally, in order to simulate optical bias that depends on several factors (e.g., angle between the thin section and the twin lamella, width and spacing of twin lamellae, extension of lamellae across grains), some twinned planes were randomly misclassified into untwinned planes. For a horizontal thin section with 100 randomly oriented grains (i.e 300 e-twin planes), and considering that a twin 
plane lying at an angle of $30^{\circ}$ or less to the thin section cannot be measured using a classical U-Stage

348 hence can be misclassified as untwinned, we estimated that the percentage of potentially optically

349 biased twin planes is always lower than $11 \%$ with an average of $6 \%$ (Fig. 6).

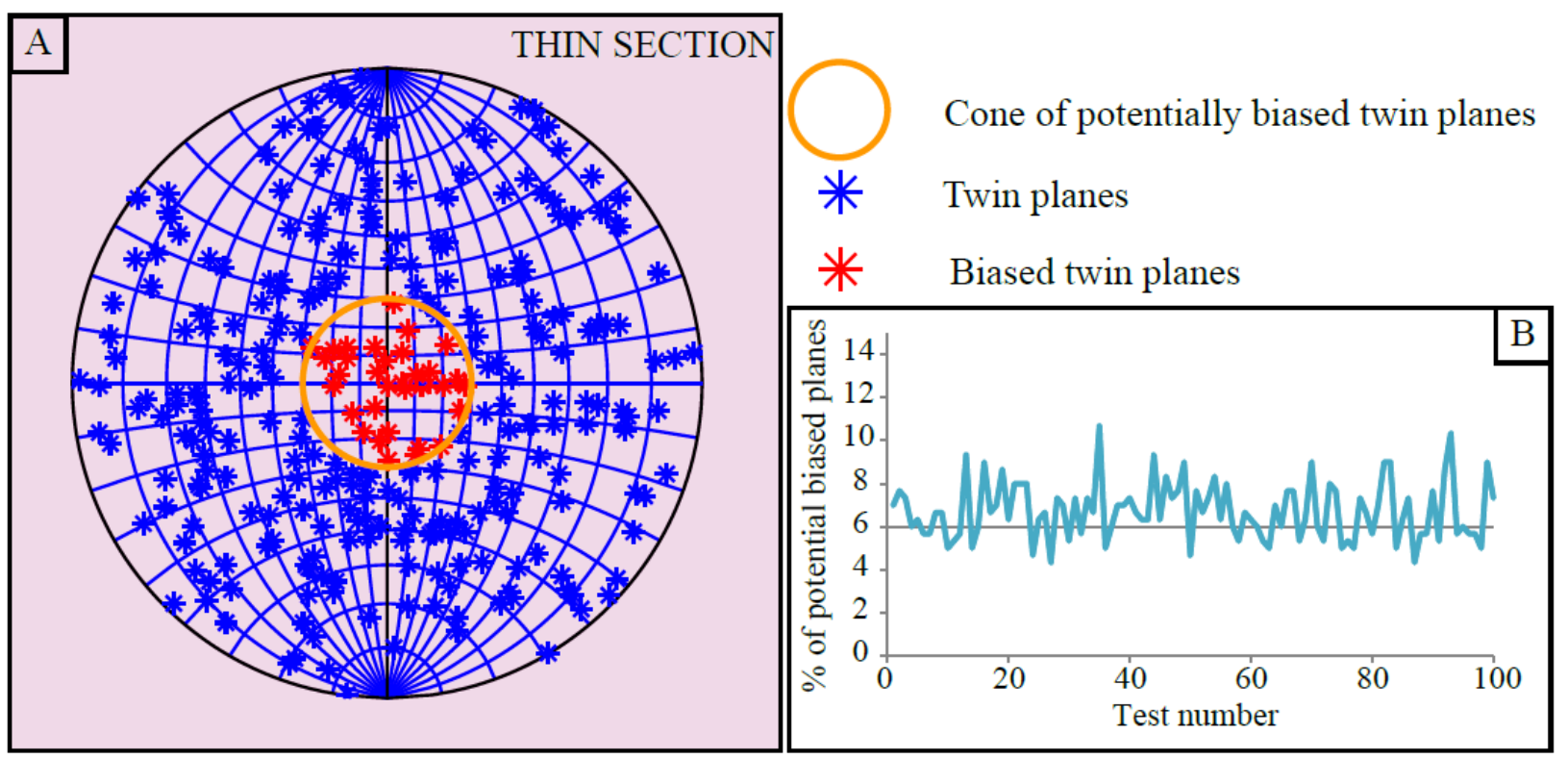

Figure 6: Evaluation of optical measurement bias related to inaccurate observations of twin planes. A) For a synthetic sample of 100 grains and 300 e-twin planes, and a horizontal thin section, the orange circle represents the cone of potentially biased poles to e-twin planes lying at less than $30^{\circ}$ of the thin section plane. B) Variation of the percentage of potentially biased e-twin planes (lying at less than $30^{\circ}$ of a virtual thin section plane cutting across a random distribution of twin planes) within various tests (100 synthetic datasets have been drawn and tested).

Following Yamaji (2015a, b), we further considered a maximum percentage of misclassified untwinned planes of $20-25 \%$ in order to also account for the other potential sources of heterogeneity at the scale of the virtual aggregate. Note however that the weight of this bias on the quality of the result of stress inversion may be highly variable. For instance, if a randomly misclassified untwinned plane is close to the optimal position for a potential twin plane to twin regarding the stress criterion, it will receive a high resolved shear stress. Then it will appear as an incompatible untwinned plane incorporated in the solution even for very low percentages of twinned planes to explain. This will greatly influence the penalization function and hence the quality of the solution. This abnormal plane is seen as an error by the software and the penalization function is strongly impacted, which could lead to discard the stress tensor from the acceptable solutions. 

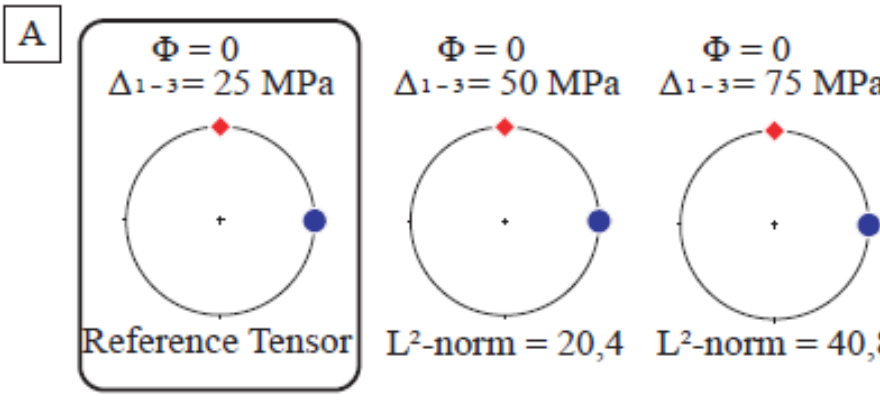

$\mathrm{L}^{2}$-norm $=20,4$

$\mathrm{L}^{2}$-norm $=40,8$
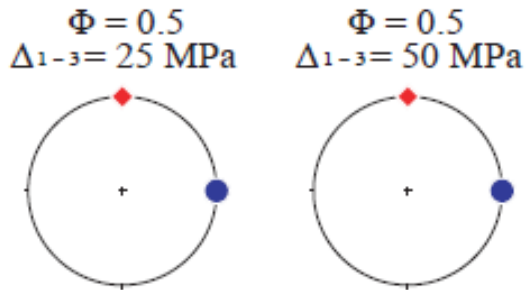

$\Phi=0.5$
$\Delta_{1-3}=75 \mathrm{MPa}$

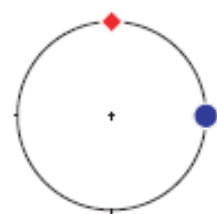

$\mathrm{L}^{2}$-norm $=10.2$

$$
\Phi=1
$$

$\Delta{ }_{1-3}=25 \mathrm{MPa}$

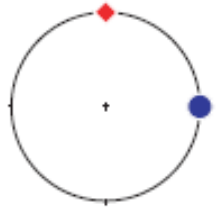

$\mathrm{L}^{2}$-norm $=20.4$

$$
\Phi=0
$$

$\Delta{ }_{1-3}=25 \mathrm{MPa}$

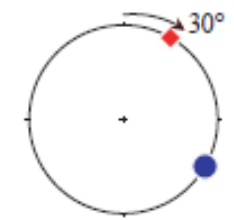

$\mathrm{L}^{2}$-norm $=17.7$

$\Delta \mathrm{1-3}=50 \mathrm{MPa}$

$\Phi=1$
$\Delta_{1-3}=75 \mathrm{MPa}$
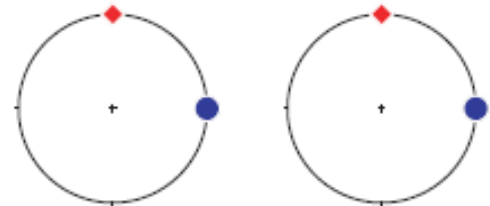

$\mathrm{L}^{2}$-norm $=35.4$

$\mathrm{L}^{2}$-norm $=54$

$\Phi=0$
$\Delta_{1-3}=25 \mathrm{MPa}$

$\Phi=0$
$\Delta_{1-3}=25 \mathrm{MPa}$

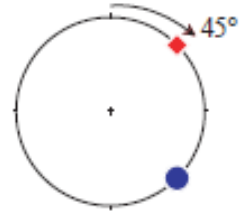

$\mathrm{L}^{2}$-norm $=25$

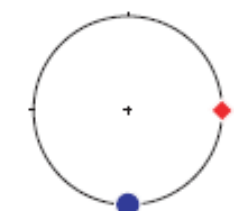

$\mathrm{L}^{2}$-norm $=35.4$
B
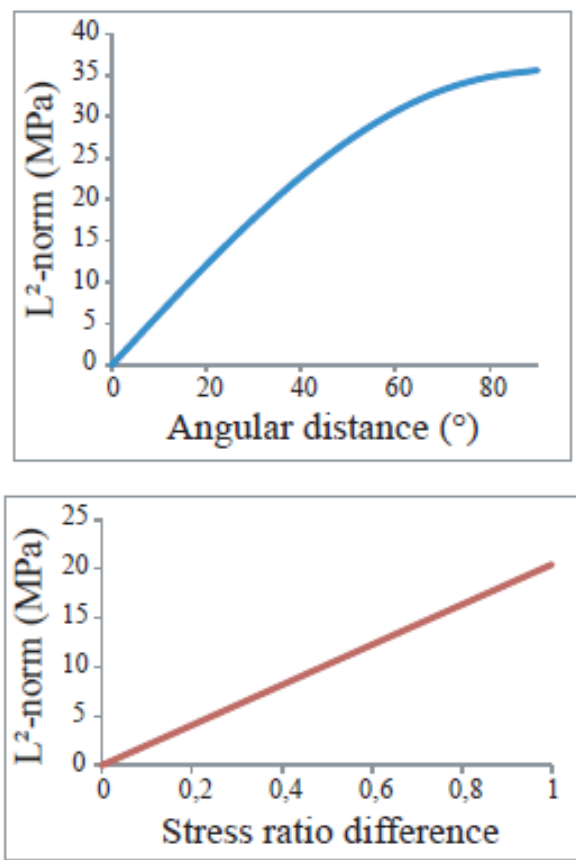

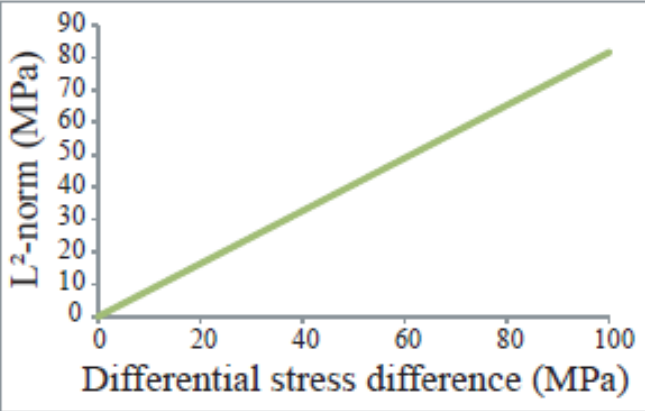

$\bullet \sigma_{1}$ axis

$\sigma$ axis

Figure 7: Evolution of the $\mathrm{L}^{2}$-norm value against variations of stress tensor parameters. A) Illustration of different tensor configurations and resulting $\mathrm{L}^{2}$-norm value. B) Sensitivity of $\mathrm{L}^{2}$-norm value to stress tensor parameters (principal stress orientation, stress ratio and differential stress).

It is necessary to efficiently compare the 5 parameter stress tensors resulting from the inversion (called "solution tensor") to the initially applied tensor (called "applied tensor"), in order to determine the accuracy of the inversion method. Peeters et al. (2009) propose different kinds of distance calculations between 5 parameter tensors. The $\mathrm{L}^{2}$-norm was chosen for our purpose, because it is the most robust for all tensor configurations: 
Pascal (MPa). Note that this equation strongly resembles the one used by Yamaji $(2015 \mathrm{~b}, \S 3.5)$ and differs only by a factor of $\sqrt{2 / 3}$. The equation can be written as a measure between tensors, $\sigma^{\mathrm{A}}$ and $371 \quad \sigma^{\mathrm{B}}$ :

$$
L^{2}-\operatorname{norm}(A, B)=\sqrt{\left(\sigma^{A}-\sigma^{B}\right):\left(\sigma^{A}-\sigma^{B}\right)}=\sqrt{\frac{2}{3}} d(7)
$$

where the colon denotes the double-dot product of tensors (Yamaji and Sato, 2006 ; Yamaji, 2007), and d is Yamaji's (2015b) dissimilarity measure.

Figure 7 helps visualize how the $\mathrm{L}^{2}$-norm value evolves with different configurations of tensors, hence its sensitivity to changes in $\sigma_{1}$ orientation, stress ratio and differential stress. The $\mathrm{L}^{2}$ norm value is clearly more sensitive to variations of differential stress than to variations of the stress ratio or principal stress orientations.

\subsection{Tests for calibration of the new inversion scheme}

For the first tests, one tensor is applied (so-called monophase dataset), with one grain size.

The grain size is simulated using the corresponding CRSS value. But in nature it is very rare to have a sample with just one grain size. To fit with reality, other tests have been carried out with two distinct grain sizes, but also with a dispersion around the two mean grain sizes (pseudo-Gaussian distribution of grain sizes associated to different CRSS values also following a pseudo-Gaussian distribution).

Taking into account the $10 \mathrm{MPa}$ CRSS value commonly adopted for grains of size of about $300 \mu \mathrm{m}$ and deformed at 3\% (see Lacombe, 2010), it has been decided to test a distribution of grain sizes as corresponding to a distribution of CRSS values centered on $10 \mathrm{MPa}$, with $60 \%$ of grains with a CRSS 
of the technique to slight variations in CRSS value or grain size within a defined grain size class.

Biases are also incorporated in order to simulate the natural heterogeneities of a rock sample as well as optical measurement mistakes due to the use of a U-stage. To incorporate heterogeneities, the program will randomly change a percentage of twinned planes (fixed by the user) into untwinned planes.

In a second part, CSIT-2 is tested on datasets with two applied tensors and the same scheme is carried out in order to determine the uncertainties in the results.

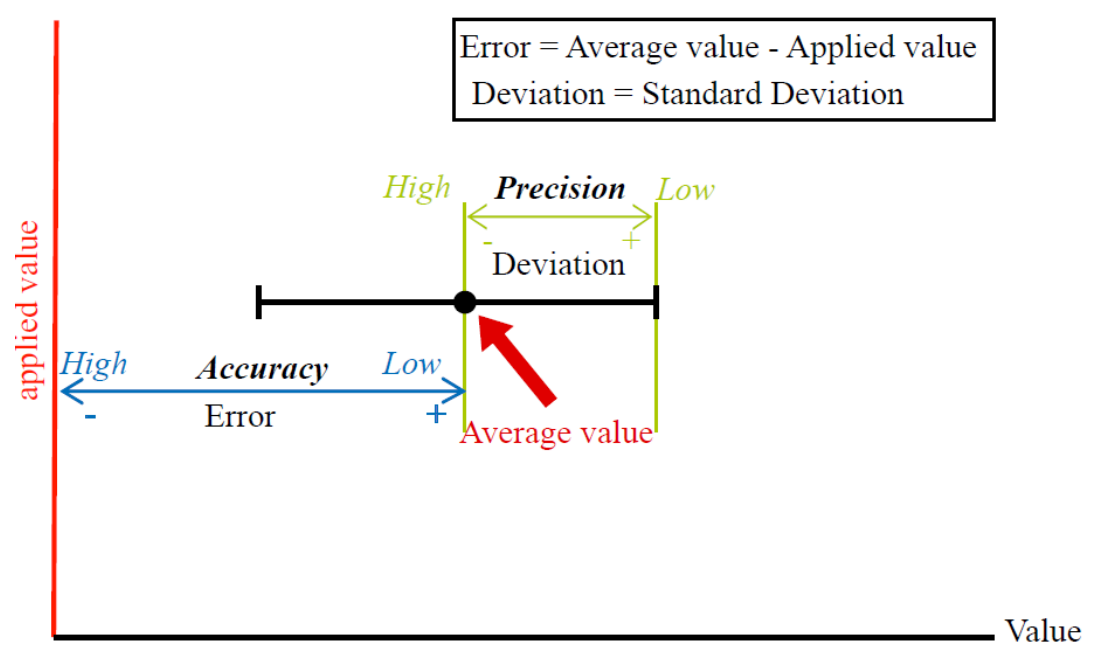

Figure 8: Definition of the terms "deviation", "error", "accuracy" and "precision".

In order to quantify and discuss the results obtained using this new technique, several tests on each configuration have been carried out in order to calculate the average value for each parameter of the deviatoric tensor (orientations, stress ratio and differential stress, Table 2 and 3). About 20-30 tests were necessary to get the stability of the solution for monophase datasets and 30-40 tests for 401 polyphase datasets. We define the precision of the technique as the degree to which successive tests 402 carried out under unchanged conditions yield the same results. The precision is quantified through evaluation of the variability of the determined stress parameter values around the mean values over the number of tests required to reach solution stability; it is called deviation hereinafter and is related to reproducibility of the results (Fig.8). The lower the deviation, the higher the precision. We further 406 define the accuracy of the technique by the degree of closeness of the mean reconstructed stress 407 parameter to the true applied stress parameter. The accuracy is quantified by the error between the 
mean stress parameter value and to the true applied value (Fig.8). The lower the error, the higher the 409 accuracy. Both error and deviation will be given either in absolute value or in percentage of the applied value. As a result, we infer that the maximum methodological uncertainty associated with the 411 determination of each stress parameter (i.e., when the inversion technique is applied blindly by a 412 potential user to a naturally deformed sample) expectedly corresponds to \pm (error + deviation) as 413 derived from the results on numerically generated twin datasets. The error on the stress ratio will be 414 calculated on the maximum value of the stress ratio, i.e., 1; so that we get the same error percentage 415 for a given error whatever the applied stress ratio. For example, if the error value on the stress ratio 416 is 0.1 for an applied stress ratio of 0.5 or 1 , the error percentage will be of $10 \%$ in both cases.

\subsection{Evaluation of the similarity of applied tensors}

The inversion process is expected to hardly detect and separate close tensors, because the percentage of common twinned planes can be very high in this case. For instance, the examination of the activated twinned planes, which are shared by both tensors with similar stress ratio but with $\sigma_{1}$ axes at $30^{\circ}$ to each other, shows that, for a homogeneous grain size dataset, the degree (percentage) of similarity increases with the differential stress applied and can almost reach $70 \%$ (Table. 4 ). In order to test the ability of the technique to detect and separate close superimposed stress tensors, two stress tensors with a deviation of $30^{\circ}$ of the $\sigma_{1}$ axis were applied. In fact in tectonic studies, two tensors with less than $30^{\circ}$ difference in principal stress orientations may be considered as similar because of cumulated inaccuracies on sample orientations and thin section making, measurement bias as well as possible local natural reorientation of stress tensors in rocks. We also tested superimposed stress tensors with perpendicular horizontal $\sigma_{1}$ axes (N-S and E-W) as well as stress tensors differing by permutations of $\sigma_{2}$ and $\sigma_{3}$ stresses (which is a frequent situation encountered in regional paleostress reconstructions). 


\begin{tabular}{|c|c|c|c|c|c|c|c|}
\hline Configuration & Tensor & $\sigma_{1}$ & $\sigma_{2}$ & $\sigma_{3}$ & $\Phi$ & $\Delta_{1-3}(\mathrm{MPa})$ & Shared twinned planes (\%) \\
\hline \multirow{2}{*}{1} & T1 & $180 / 0$ & 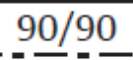 & $270 / 0$ & $\underline{0,5}$ & 35 & \multirow{2}{*}{53,8} \\
\hline & $\overline{\mathrm{T}} 2$ & $30 / 0$ & $120 / 90$ & $300 / 0$ & $\overline{0}, \overline{5}$ & 35 & \\
\hline \multirow{2}{*}{2} & T3 & $180 / 0$ & 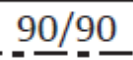 & $270 / 0$ & $\underline{0,5}$ & 50 & \multirow{2}{*}{55,9} \\
\hline & $\overline{\mathrm{T}} 4$ & $30 / 0$ & $120 / 90$ & $300 / 0$ & 0,5 & 50 & \\
\hline \multirow{2}{*}{3} & T5 & $180 / 0$ & $90 / 90$ & 27으므. & 0,5 & 75 & \multirow{2}{*}{60,5} \\
\hline & $\overline{\mathrm{T}} 6$ & $30 / 0$ & $120 / 90$ & $300 / 0$ & 0,5 & 75 & \\
\hline \multirow{2}{*}{4} & T7. & $180 / 0$ & $90 / 90$ & $270 / 0$ & $\underline{0}$ & 35 & \multirow{2}{*}{47,1} \\
\hline & $\mathrm{T} 2$ & $30 / 0$ & $120 / 90$ & $300 / 0$ & 0,5 & 35 & \\
\hline \multirow{2}{*}{5} & T8 & $180 / 0$ & 90으뭉 & $270 / 0$ & $\underline{0}$ & 50 & \multirow{2}{*}{62,5} \\
\hline & $\mathrm{T} 4$ & $30 / 0$ & $120 / 90$ & $300 / 0$ & 0,5 & 50 & \\
\hline \multirow{2}{*}{6} & T9 & $180 / 0$ & $90 / 90$ & $270 / 0$ & - & 75 & \multirow{2}{*}{67,7} \\
\hline & $\mathrm{T} 6$ & $30 / 0$ & $120 / 90$ & $300 / 0$ & 0,5 & 75 & \\
\hline \multirow{2}{*}{7} & T3. & 180/0 & 90/90 & $270 / 0$ & $\underline{0,5}$. & 50 & \multirow{2}{*}{69,6} \\
\hline & $\mathrm{T} 2$ & $30 / 0$ & $120 / 90$ & $300 / 0$ & 0,5 & 35 & \\
\hline \multirow{2}{*}{8} & T3. & $180 / 0$ & $90 / 90$ & $270 / 0$ & $\underline{0,5}$ & 50 & \multirow{2}{*}{53,7} \\
\hline & T6 & $30 / 0$ & $120 / 90$ & $300 / 0$ & 0,5 & 75 & \\
\hline \multirow{2}{*}{9} & T1 & $180 / 0$ & $90 / 90$ & $270 / 0$ & 0,5 & 35 & \multirow{2}{*}{30,4} \\
\hline & $\mathrm{T} 10$ & $180 / 0$ & $90 / 0$ & $270 / 90$ & 0,5 & 35 & \\
\hline \multirow{2}{*}{10} & T3 & $180 / 0$ & $90 / 90$ & $270 / 0$ & 0,5 & 50 & \multirow{2}{*}{52,6} \\
\hline & T11 & $180 / 0$ & $90 / 0$ & $270 / 90$ & 0,5 & 50 & \\
\hline \multirow{2}{*}{11} & T5 & $180 / 0$ & $90 / 90$ & $270 / 0$ & 0,5 & 75 & \multirow{2}{*}{66,2} \\
\hline & $\mathrm{T} 12$ & $180 / 0$ & $90 / 0$ & $270 / 90$ & 0,5 & 75 & \\
\hline
\end{tabular}

Table 4: Evaluation of the degree of similarity between applied tensors. The table reports the maximum number of twin planes which are twinned by the two applied stress tensors for various stress configurations. These numbers are derived from 100 tests for each configurations. $\Phi$ is the stress ratio and $\Delta 1-3$ is the differential stress. Stress axis orientations are given in azimut/dip $\left({ }^{\circ}\right)$.

The similarity percentage is calculated by dividing the number of twinned planes shared by both tensors by the number of planes twinned by the second tensor. This parameter is not symmetrical:

433 comparing A with B or B with A. It has been decided to compare the second tensor applied with the

434 first one because the first tensor can activate all the possible twin planes (depending on the equations 4351 and 2) which is not the case for the second applied tensor (for which a strain hardening is applied).

436 The second tensor can therefore activate twinning on a lower number of twin planes than the first 437 applied tensor. It is this reason why the similarity criterion is calculated based on the second applied 438 tensor. The configuration with a permutation of $\sigma_{2}$ and $\sigma_{3}$ stress axes and the configuration with two 439 tensors the orientation of the maximum principal stresses of which differ by $30^{\circ}$ are supposed to be 440 the most complex configurations in terms of tensor detection and separation. As mentioned before, 
441 the percentage of similarities can reach 69.6\% at high differential stress (configuration 7 in table1).

442 This is the reason why the critical percentage of similarities accepted by the process of tensor selection 443 in the new CSIT-2 inversion scheme is $70 \%$ by default. We consider that this value makes it possible 444 to differentiate most of the tensors belonging to different tectonic phases even in case of large 445 differential stresses, hence large percentages of similarities.

\section{Results}

\subsection{Monophase twin dataset}

In the following part, the tests start with the simplest case, a monophase dataset with 449 homogeneous grain size and no bias. Then, in order to simulate an increasing demand for the 450 technique, this dataset is then made heterogeneous in terms of grain size, and will further incorporate 451 virtual measurement bias. The basic stress configuration involves a N-S trending $\sigma_{1}$ axis and a E-W 452 trending $\sigma_{3}$ axis.

\subsubsection{Monophase twin dataset with homogeneous grain size}

The first tests were carried out using datasets with one applied tensor and one grain size (CRSS

$455=10 \mathrm{MPa}$ for all grains). A single orientation of principal stress axes was tested, but for this 456 configuration, different stress ratios $(0,0.5$ and 1) and differential stresses (25 to $75 \mathrm{MPa})$ were 457 applied (configurations M1, M2, M3, M4, M5 and M6 in table 2).

Results from the inversion process show that the orientation of principal stress axes, stress ratio and differential stress are well reconstructed (Figure 9). A slight discrepancy is observed for differential stress magnitudes; the error however remains lower than $2.2 \mathrm{MPa}(3 \%$, Figure 9). 


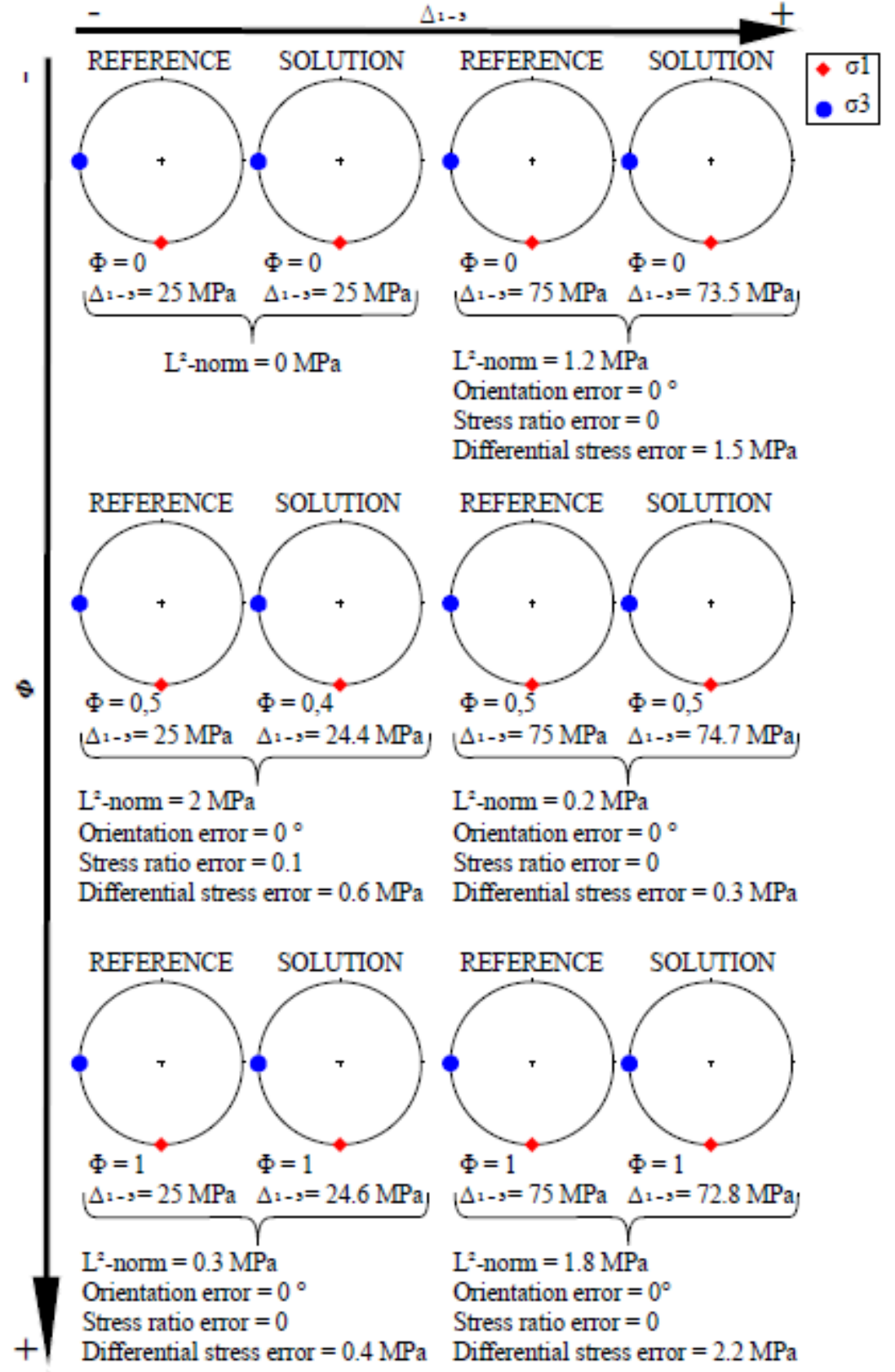

Figure 9: Results of inversion of numerically-generated monophase datasets with homogeneous grain size (configurations M1 to M6 in table 2). $\Phi$ is the stress ratio and $\Delta_{1-3}$ is the differential stress in MPa. Total error is $\mathrm{L}^{2}$-norm. The error on the orientation is calculated using the scalar product between $\sigma_{1}$ axes of applied and solution tensors.

Additional tests were carried out following the same scheme but with a slight dispersion 462 around the prevailing grain size (Gaussian distribution around a CRSS of $10 \mathrm{MPa}$, configurations M9, $463 \mathrm{M} 10$ and M12 in table 2). Results show that for the error ( $\mathrm{L}^{2}$-norm) between the applied tensors and 464 the solution tensors increases with the applied differential stress but remains very low and does not 465 exceed $6 \mathrm{MPa}$ (Fig. 10). The error on the orientation of principal stress axes is on average lower than $4664^{\circ}$ with a deviation of $\pm 2^{\circ}$ (Fig. 10). For the stress ratio and the differential stress values the error is 467 close to zero with a very low deviation (Fig.10). 


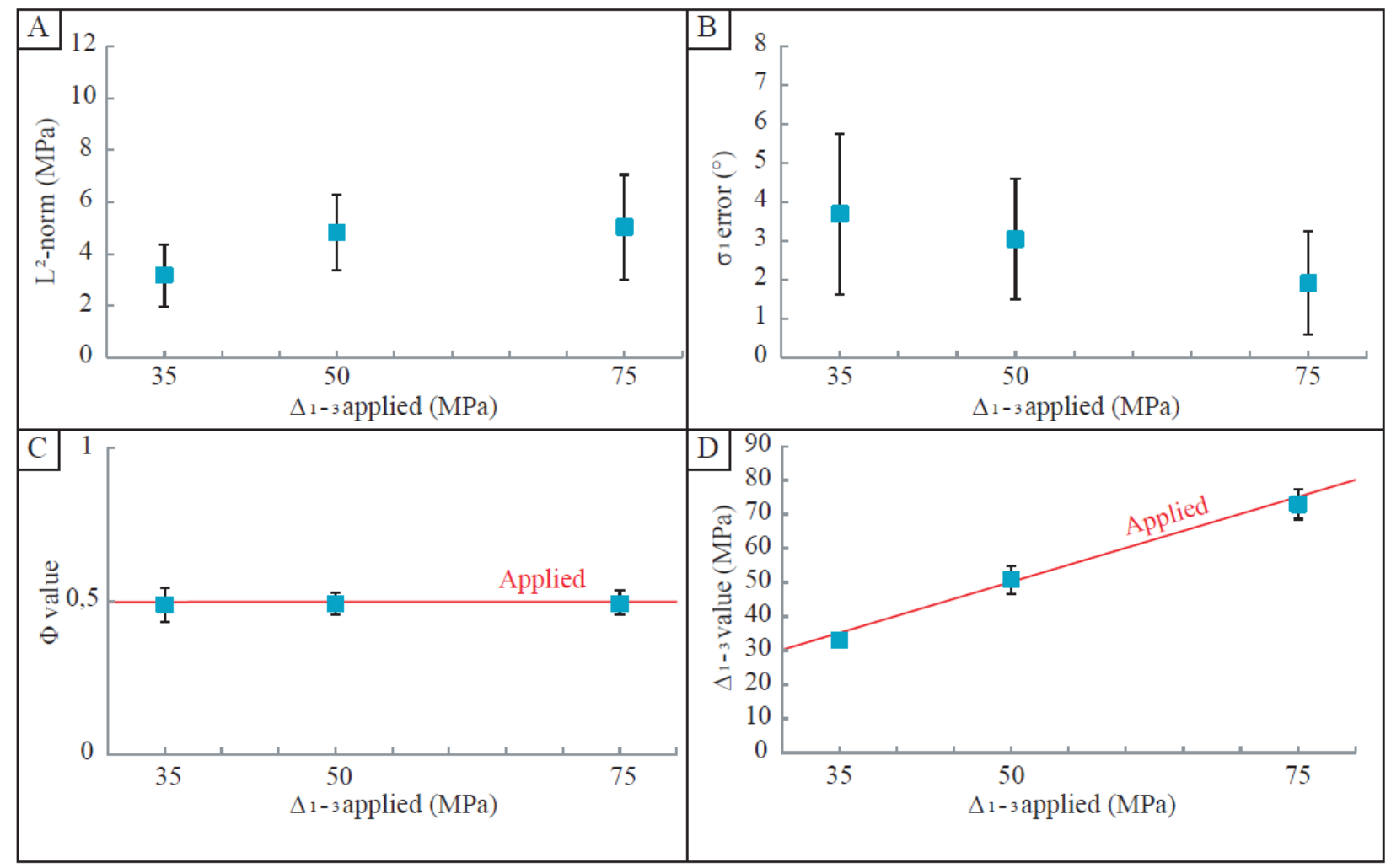

Figure 10: Inversion results of numerically-generated monophase datasets M9, M10 and M12. The graphs show $\mathrm{A}$ ) the results of the $\mathrm{L}^{2}$-norm error and deviation between the applied tensor and the solution tensor, B) the error and deviation on the $\sigma_{1}$ orientations, C) the deviation of stress ratio values with the mean value and $\mathrm{D}$ ) the deviation differential stress values with the mean value. The red line represents the reference (applied) stress ratio and differential stress values.

\subsubsection{Monophase twin dataset with heterogeneous grain size}

Datasets with virtually heterogeneous grain sizes are simulated using two separate CRSS values. These values are $5 \mathrm{MPa}(2 / 3$ of grains) and $15 \mathrm{MPa}$ (1/3 of grains) (configurations $\mathrm{M} 7$ and M8 in table 2). However, the $10 \mathrm{MPa}$ value of the CRSS was still blindly considered for the inversion process, in order to test the robustness of the technique with respect to significantly varying grain sizes in an aggregate. Figures 11A and B show the application of inversion process for applied tensors with variable differential stress of $50 \mathrm{MPa}$ and $75 \mathrm{MPa}$ and two perfectly distinct grain sizes. The solution tensor is nearly similar to the applied tensor for $50 \mathrm{MPa}$, whereas it differs more importantly from the applied tensor with $75 \mathrm{MPa}$, especially in terms of differential stress. According to Yamaji

477 (2015), there is a loss of information on the differential stress value given by the inversion if this value is greater than 50-100 MPa. For these applied tensors, we further tested a constant virtual CRSS 


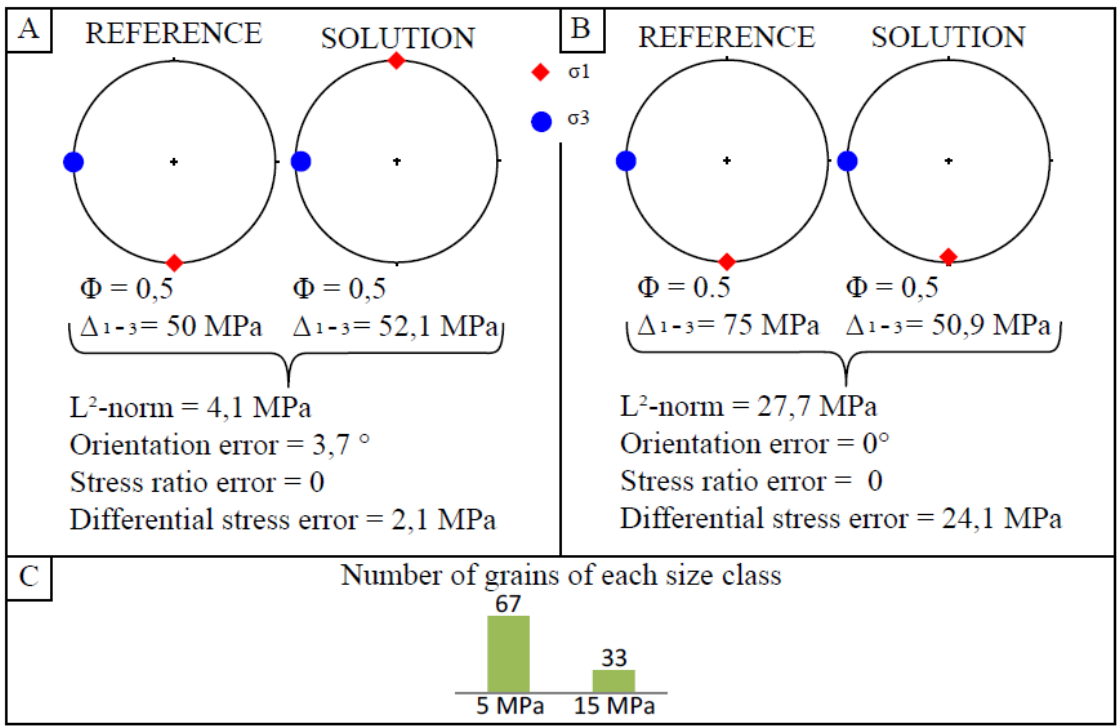

Figure 11: Inversion results of numerically-generatedmonophase datasets A) M7 and B) M8 (Table 2). C) Histogram showing the number of grains of each size class used during simulation.

Datasets with a slight (pseudo-Gaussian type) dispersion within the two considered grain size classes are further considered (configurations M12, M13 and M14 in table 2). The error ( $\mathrm{L}^{2}$-norm) is lower than $5 \mathrm{MPa}$ but increases with the differential stress, reaching the value of $8 \mathrm{MPa}$. There is therefore a loss of information about the whole 5 parameters of the tensor around $75 \mathrm{MPa}$ of differential stress (Fig. 12A). In details, regarding the principal stress orientations and stress ratio, they are well found, and the related errors do not increase with increasing applied differential stress (Fig. 12B and C). The error on the differential stress is of $0-1 \mathrm{MPa}(1-3 \%$ of the applied value) below $50 \mathrm{MPa}$ and of $3 \mathrm{MPa}(4 \%)$ at $75 \mathrm{MPa}$ (Fig. 11). The deviation is of $\pm 1-2 \mathrm{MPa}(4-5 \%)$ for differential stress below $50 \mathrm{MPa}$ but increases for differential stress at $75 \mathrm{MPa}( \pm 7 \mathrm{MPa} / 9 \%)$. Compared with the previous results dealing with a single grain size class, both deviations and errors are higher, especially on the differential stress value. This is mainly due to the grain size dependence of the CRSS that has not been considered here since a $10 \mathrm{MPa}$ value for the CRSS has been blindly used to 
calculate differential stress irrespective of the heterogeneous grain size.

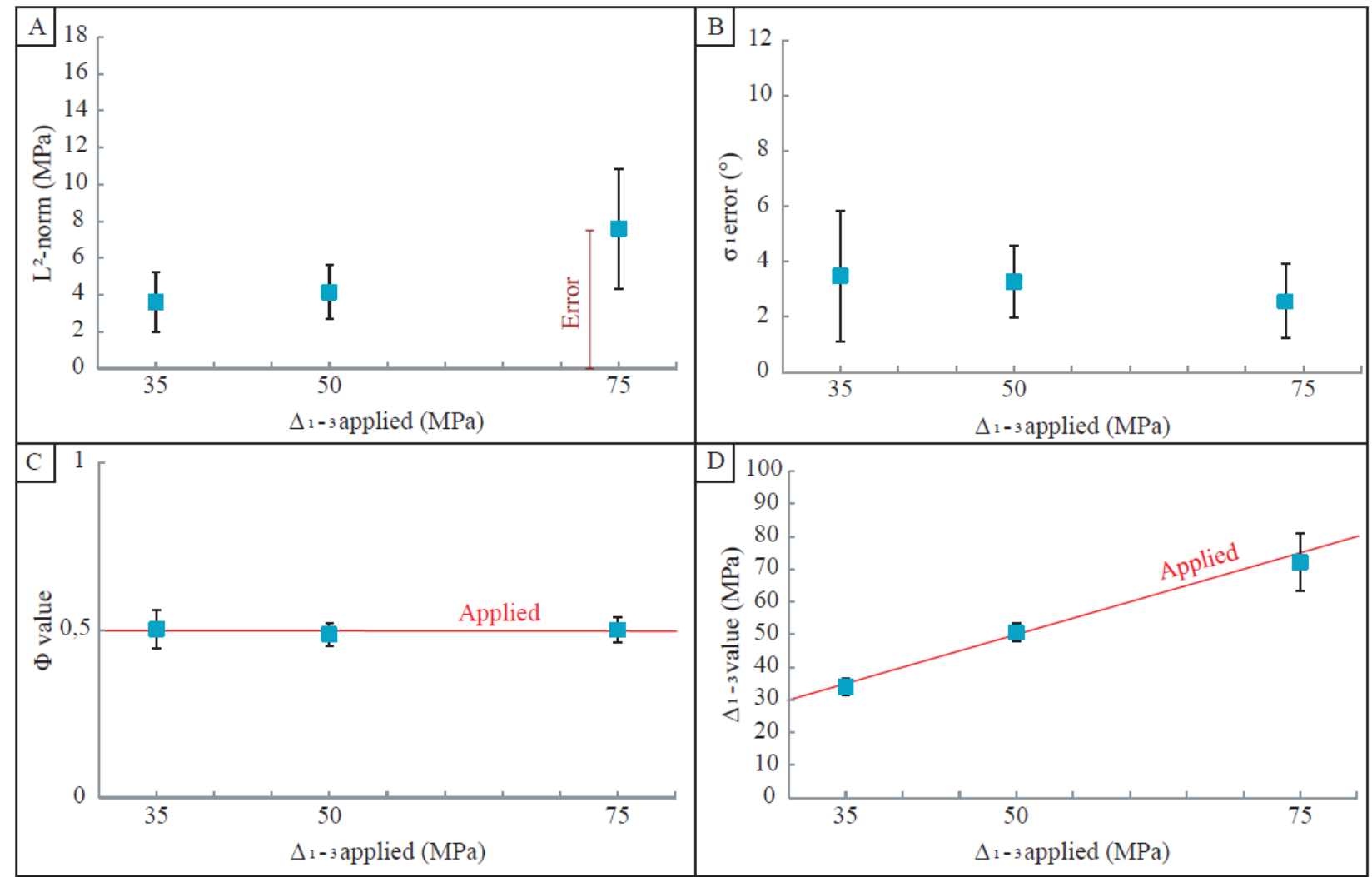

Figure 12: Inversion results of numerically-generated monophase datasets M12, M13 and M14 (Table 2). A, B, C and D same key as in figure 10.

Separating the datasets into virtual homogeneous grain size classes strongly improves the results as shown by the tests on datasets with homogeneous grain size. Thus, as discussed later, it is of first importance to carry out inversion only after the dataset has been divided into nearly homogeneous grain size classes, each corresponding to a given value of the CRSS. This is the reason why all the tests shown hereinafter are only performed on datasets corresponding to a homogeneous grain size (pseudo-Gaussian dispersion around a single CRSS value of $10 \mathrm{MPa}$ ).

\subsubsection{Monophase twin dataset with homogeneous grain size and optical bias}

In order to test the effect of an increasing bias, $10 \%$ or $25 \%$ of twinned planes have been randomly changed into untwinned planes. Results are presented in figure 13. Each point corresponds to the result of 20-30 tests. A stress ratio of 0.5 and a differential stress of $50 \mathrm{MPa}$ are imposed 507 (configurations M15 and M16 in table 2). The error ( $\mathrm{L}^{2}$-norm) for $10 \%$ or $25 \%$ of incorporated bias 
are very similar (Fig. 13A) but with a larger deviation for the results with $25 \%$ of bias. If the

509 parameters of the deviatoric stress tensor are considered one by one, the principal stress orientations,

510 stress ratio and differential stress are very similar to those of the applied tensor. Thereby, the error on

$511 \sigma_{1}$ orientation is of $3^{\circ}$ with an deviation of $\pm 2^{\circ}$ (Fig. 13B), there is a negligible error $(<0.01)$ for the

512 stress ratio but an deviation of less than $\pm 0.1(10 \%)$ (Fig 13C), and a very slight error $(<2 \mathrm{MPa})$ on

513 differential stress values with an deviation of about $10 \%$ or less (Fig. 13D).

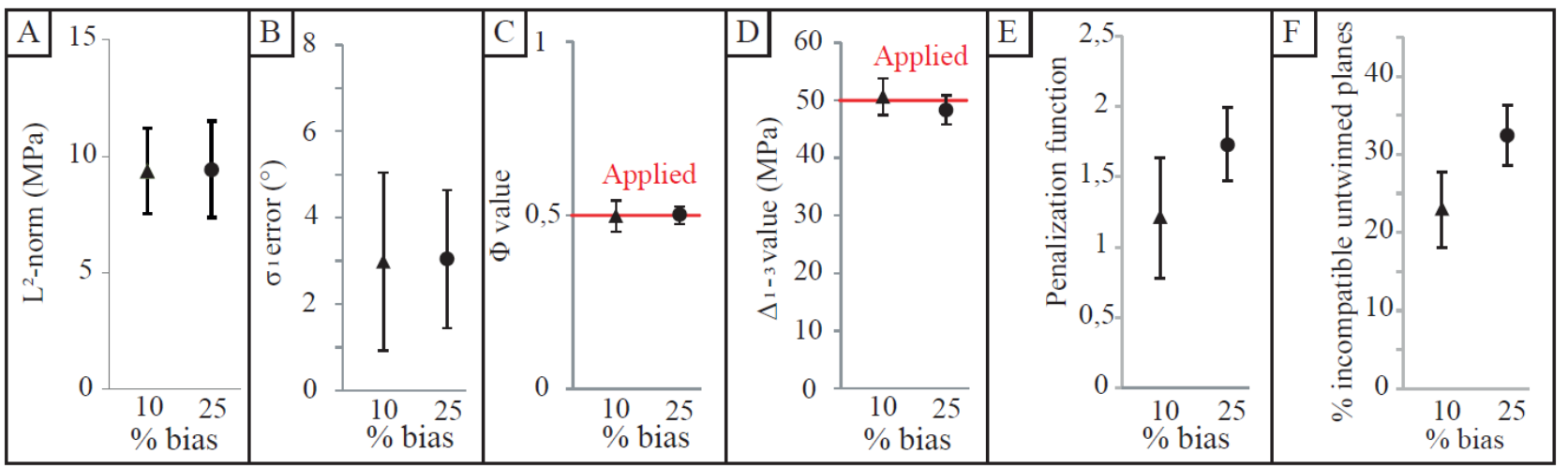

Figure 13: Inversion results of numerically-generated monophase datasets M15 and M16 (Table 2). $\mathrm{A}, \mathrm{B}, \mathrm{C}$ and $\mathrm{D}$ same key as in figure 10.

Figure $13 \mathrm{E}$ and $\mathrm{F}$ show that with $10 \%$ and $25 \%$ bias, a solution tensor with a low error $\left(\mathrm{L}^{2}\right.$ -

norm) is found to the cost of an increase of the penalization function and also of the incorporation of

516 incompatible untwinned planes with the increase of the incorporation of bias. For the tests with $25 \%$

517 of bias, the solution have been determined on the basis of the breaks in slope of curves as previously

518 discussed, but the values for the penalization function and the percentage of incompatible untwinned

519 planes in the solution are greater than the one for $10 \%$ or even $0 \%$ of incorporated bias. This means

520 that compared to the $0 \%$ bias configuration M10 a larger tolerance threshold toward increasing

521 penalization function value for $10 \%$ or $25 \%$ of bias explains the similar values for the $\mathrm{L}^{2}$-norm.

\subsection{Polyphase calcite twin dataset}

\subsubsection{Polyphase twin dataset with homogeneous grain size, no bias and different stress ratio}

Results are shown for polyphase cases with two applied tensors (configurations B18 then B21, 
on $10 \mathrm{MPa})$. The first applied tensor is a N-S compression with an E-W extensional part $\left(\sigma_{1}\right.$ : N180$0 ; \sigma_{3}: \mathrm{N} 270-0$ ) with a stress ratio of 0.3 . The second applied tensor is turned of $30^{\circ}$ between $\sigma_{1}$ axes

$528\left(\sigma_{1}: \mathrm{N} 30-0 ; \sigma_{3}: \mathrm{N} 300-0\right)$ with a stress ratio of 0.7 . These tensors have been applied on twin data for an increasing differential stress $(35,50$ and $75 \mathrm{MPa})$ (Fig. 14A). The results of the CSIT-2 inversion on theses 3 configurations show that there is a loss of information with increasing differential stress (Fig. 14B). Figure 14C shows that the orientation of the two applied tensors are well recovered with an error of $4-7^{\circ}$ (and a deviation of $2-3^{\circ}$ ) for both tensors. The error on the stress ratio for the first 533 tensor is negligible $(<0.1)$ with a deviation of \pm 0.03-0.05 $( \pm 3-5 \%)$ (Fig. 14D). For the second tensor, 534 the error on the stress ratio is of $0.06-0.13(6-13 \%)$ with a deviation of $\pm 0.04-0.06( \pm 4-6 \%)$. The differential stress is well retrieved with an error of $0.05-2 \mathrm{MPa}(0.15-6.5 \%)$ with a deviation of $\pm 1-2$ $\mathrm{MPa}( \pm 2.9-5.7 \%)$ for low applied differential stress $(<50 \mathrm{MPa})$ and of $\pm 5-6 \mathrm{MPa}( \pm 6-8 \%)$ for an applied differential stress of $75 \mathrm{MPa}$ (Fig. 14E). These results show that CSIT-2 is able to identify and separate superimposed stress tensors with very different stress ratios. Figure 14D shows that with increasing differential stress there is no loss of accuracy but a loss in the precision of the results.

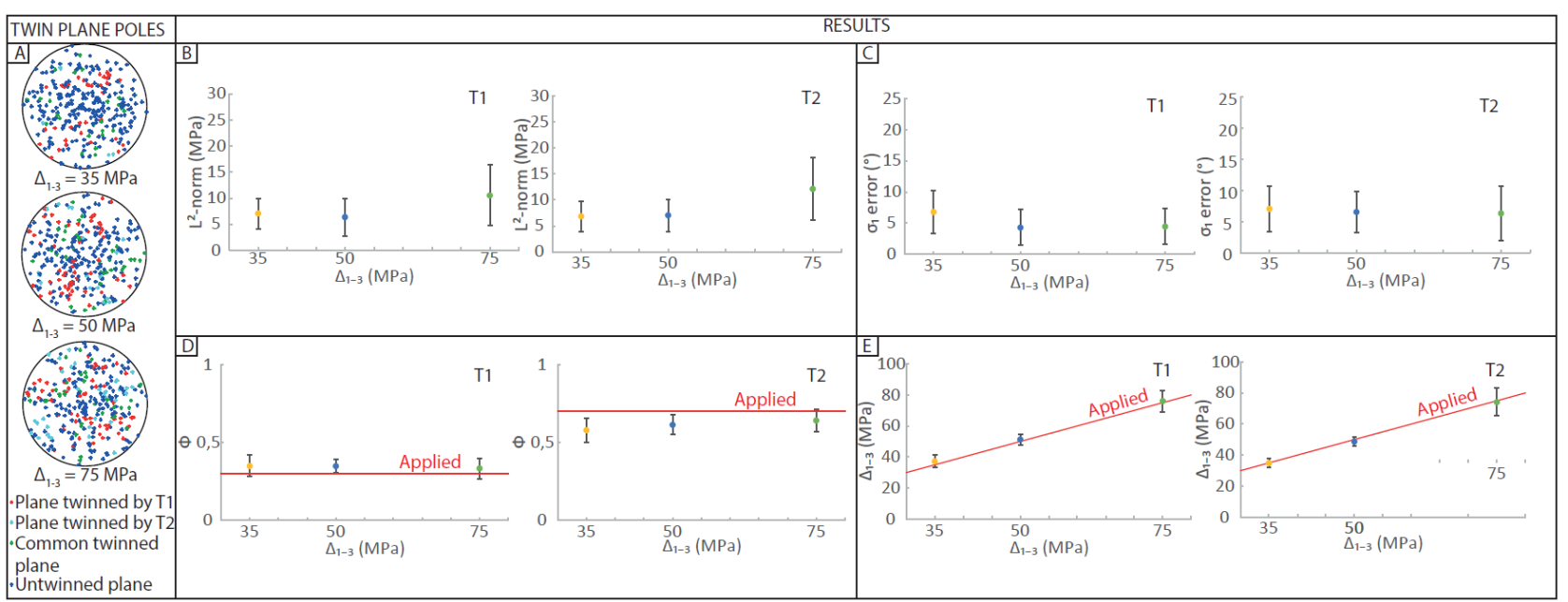

Figure 14: Inversion results of numerically-generated polyphase datasets B18 then B21, B19 then B22 and B20 then B23 configurations (Table 3). A) Wulff stereo-diagrams (lower hemisphere) of twin plane poles generated for the three configurations described previously. The graphs show the results of the inversion with $\mathrm{B}$ ) the results of the $\mathrm{L}^{2}$-norm error and deviation between the applied tensor and the solution tensor, C) the error and deviation on the $\sigma_{1}$ orientations, D) the deviation of stress ratio values with the mean value and $\mathrm{E}$ ) the deviation differential stress values with the mean value. The red line represents the reference (applied) stress ratio and differential stress values. 
Results for polyphase cases with a difference of $30^{\circ}$ between $\sigma_{1}$ axes of both tensors are summarized in figure 15 (configurations B1 then B3 and B2 then B4 in table 3). The distribution of grain size (i.e. CRSS value) is a pseudo-Gaussian, centered on a CRSS value of $10 \mathrm{MPa}$. The stress ratio and the differential stress are the same for both tensors, that is, $\Phi=0.5$ and $\Delta_{1-3}=50 \mathrm{MPa}$.

545 Figure 15B shows that, for the first tensor applied the error is of 9-11 MPa for the $\mathrm{L}^{2}$-norm with 10 or $25 \%$ of optical bias. Adding optical bias (10-25\%) has almost no impact on the differential stress parameter. For the second tensor, the error $\left(\mathrm{L}^{2}\right.$-norm $)$ is about $10 \mathrm{MPa}$. The first tensor has an error of $6^{\circ}$ on the orientation of principal stress axes with a deviation of $\pm 2-3^{\circ}$ (Fig. 15C). The second tensors has an error of $7-8^{\circ}$ on the principal stress orientations with a deviation of $\pm 2-3^{\circ}$ (Fig. $15 \mathrm{C}$ ).

The accuracy for the second tensor is slightly lower than for the first one. The error on the stress ratio parameter is of $0-0.03(3 \%)$ with a deviation of $\pm 0.03-0.09(3-9 \%)$ for both tensors (Fig. 15D). The error on the differential stress parameter is of $1-7 \mathrm{MPa}(2-14 \%)$ with a deviation of $\pm 5 \mathrm{MPa}(10 \%)$ for both tensors (Fig. 15E). The different parameters of the stress tensors are fairly well estimated.

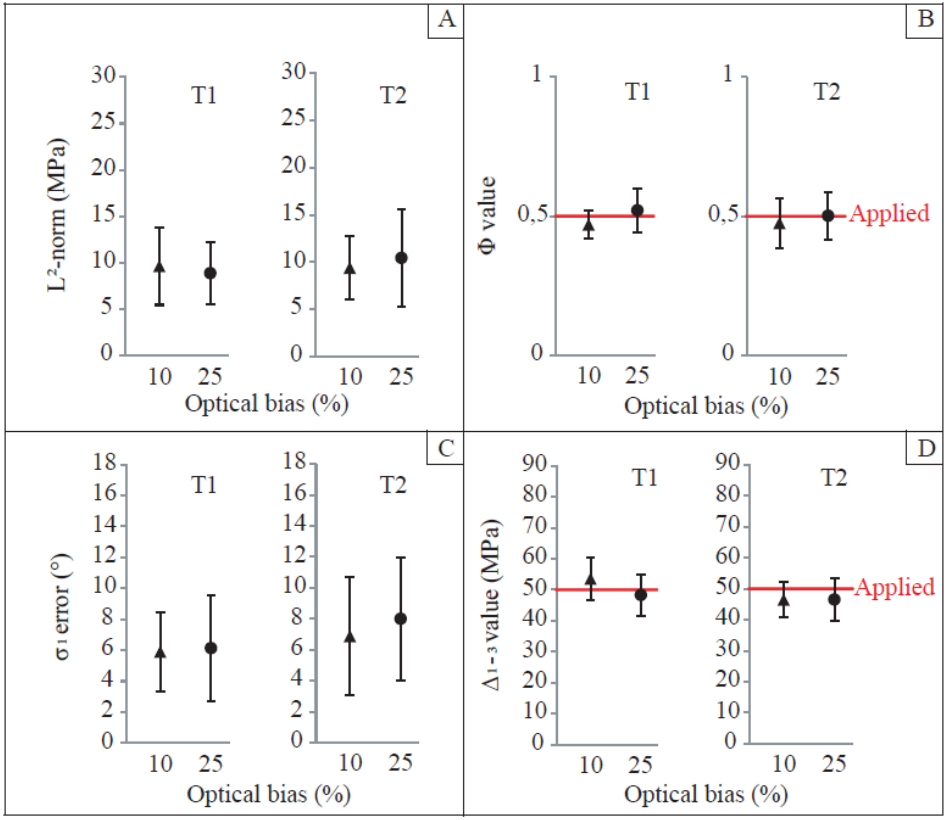

Figure 15: Inversion results of numerically-generated polyphase datasets applying B1 and B3 configurations then $\mathrm{B} 2$ and B4 configurations (Table 3). A) the results of the $\mathrm{L}^{2}$-norm error and deviation between the applied tensor and the solution tensor, B) the error and deviation on the $\sigma_{1}$ orientations, C) the deviation of stress ratio values with the mean value and D) the deviation differential stress values with the mean value. The red line represents the reference (applied) stress ratio and differential stress values. 
are accepted. Thus a value of $10 \%$ of bias will only be tested hereinafter.

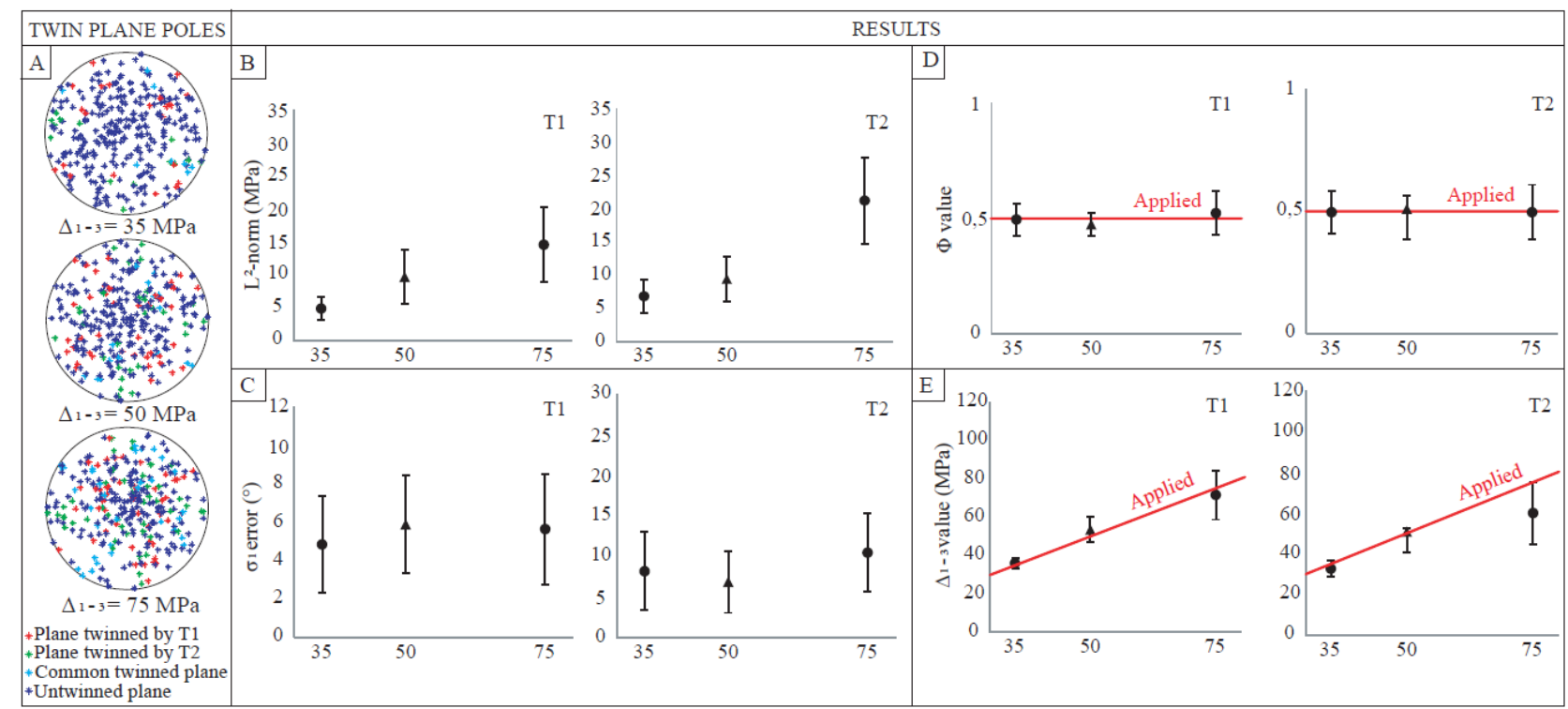

Figure 16 : Inversion results of numerically-generated polyphase datasets applying B5 then B7, B1 then B3 and B6 then B8 configurations (Table 3). A, B, C, D and E same key as in figure 14.

Having in mind the expected loss of information related to high applied differential stress, the influence of the differential stress on the result of the inversion process was then tested. The applied parameters are two stress tensors with $30^{\circ}$ of difference in orientation of the $\sigma_{1}$ axes, a stress ratio of 0.5 and $10 \%$ of optical bias (configurations B5 then B7, B1 then B3 and B6 then B8 in table 3). Three differential stresses are tested: 35,50 and $75 \mathrm{MPa}$. Results show that the error increases with the applied differential stress (Fig. 16B). The error on the orientation of $\sigma_{1}$ (Fig. 16C) is of less than $6^{\circ}$

564 (with a deviation of $\pm 2^{\circ}$ ) for the first tensor and of $10^{\circ}$ (with a deviation of $\pm 5^{\circ}$ ) for the second tensor.

565 The error on the stress ratio value is close to zero $\left(<10^{-1}\right)$ for both tensors and the deviation is of \pm $0.1(10 \%)$ (Fig. 16D). The error on the differential stress value is of 1-3 MPa (3-8\%) for the first tensor and the deviation is of 2-10 MPa (6-13\%) (Fig. 16E). For the second tensor with an applied differential stress lower or equal to $50 \mathrm{MPa}$ the error is of 2-3 $\mathrm{MPa}(7 \%)$ with a deviation of \pm 3-5 $\mathrm{MPa}(9 \%)$ (Fig. 16E). At $75 \mathrm{MPa}$, the error increases for the second tensor. This error is of $-15 \mathrm{MPa}$ $(-20 \%)$ for a deviation of $\pm 13 \mathrm{MPa}(21 \%)$ (Fig. 16E). Looking at the different stress parameters, the 
error for the last tests with a clear underestimate of the differential stress. This last observation concurs with the results of Yamaji's (2015b) study.

The influence of the differential stress on the results of the inversion process is also tested on the following cases with two tensors with $\sigma_{1}$ and $\sigma_{3}$ axes rotated by $90^{\circ}$ (configurations $\mathrm{B} 5$ then $\mathrm{B} 12$, $\mathrm{B} 1$ then $\mathrm{B} 13$ and $\mathrm{B} 6$ then B14 in table 3). Figure 15 shows the $\mathrm{L}^{2}$-norm variation with various applied differential stress: 35,50 and $75 \mathrm{MPa}$ and a stress ratio of 0.5 for both tensors. For both tensors, a similar trend is observed with an increase of the average $\mathrm{L}^{2}$-norm value (the error) up to $75 \mathrm{MPa}$ of applied differential stress and of the deviation (Fig. 17B). For principal stress orientations (Fig. 17C), the error remains the same for both tensors and even with increasing differential stresses (of about 2$4^{\circ}$ ) with a slight deviation of $\pm 1^{\circ}$. The error on the stress ratio is the lowest (Fig. 17D), as well as for the associated deviation. Figure 17E also indicates that inversion provides a very good estimate of the differential stress until $50 \mathrm{MPa}$. There is an increase of the error between 50 and $75 \mathrm{MPa}$ for both tensors which can be estimated approximately at $-6 \mathrm{MPa}(-10 \mathrm{MPa}$ for the second tensor) with a very slight deviation of $\pm 5 \mathrm{MPa}(7 \%)$.

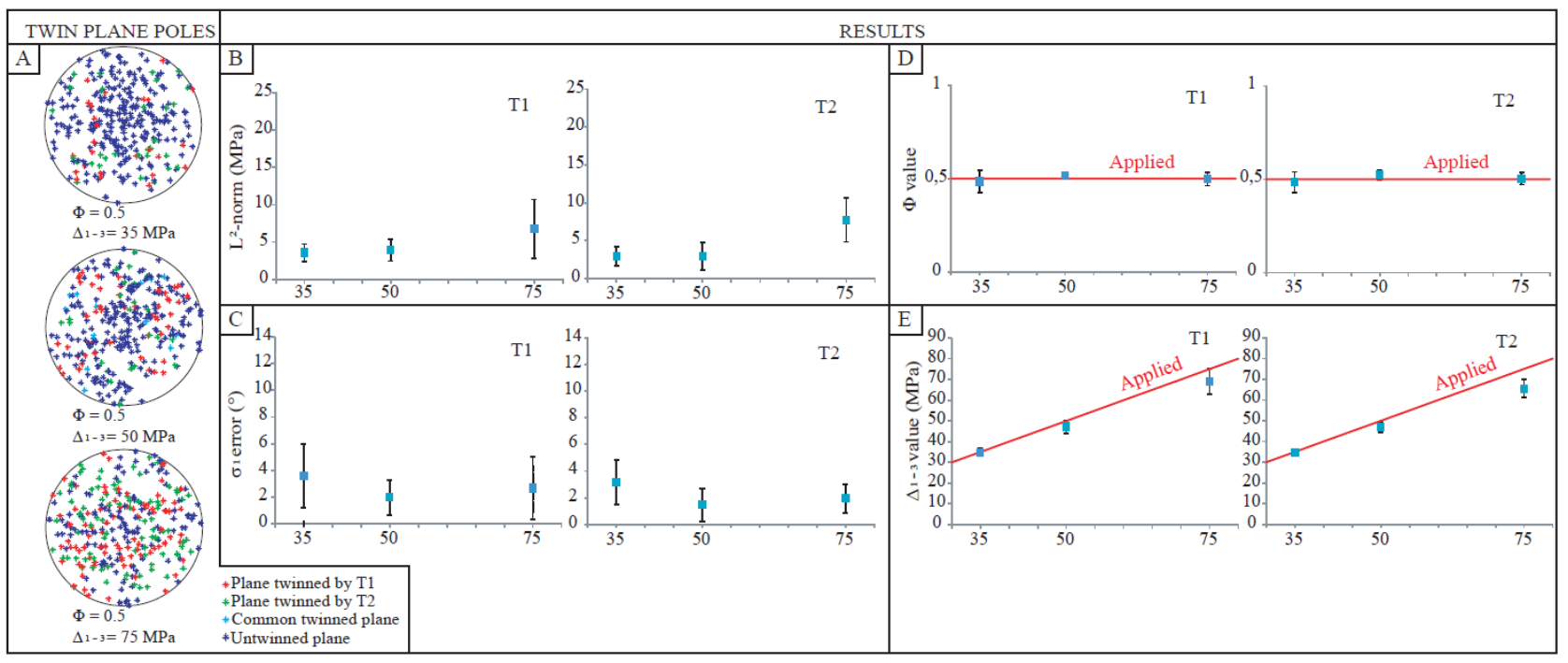

Figure 17: Inversion results of numerically-generated polyphase datasets applying B5 then B9, B1 then B10 and B6 then B11 configurations (Table 3). A, B, C, D and E same key as in figure 14. $18 \mathrm{~A})$ with similar $\sigma_{1}$ axes, stress ratio $(0.5)$ and differential stress $(35,50$ or $75 \mathrm{MPa})$ but with a 
permutation of $\sigma_{2}$ and $\sigma_{3}$ axes (configurations B5 then B12, B1 then B13 and B6 then B14 in table 3 ).

589 The error $\left(\mathrm{L}^{2}\right.$-norm) is the same for both tensors (less than $10 \mathrm{MPa}$ ) and is stable with increasing differential stress (Fig. 18B). The error on principal stress orientation is low (Fig. 18C), less than $5^{\circ}$

591 for both tensor and the deviation is about $\pm 2-3^{\circ}$. The error on the stress ratio (Fig. 18D) is decreasing with increasing differential stress and the same for the deviation. The differential stress is perfectly found with an error of $0 \mathrm{MPa}$ and a deviation of less than $\pm 5 \mathrm{MPa}$ (7\%) (Fig. 18E).

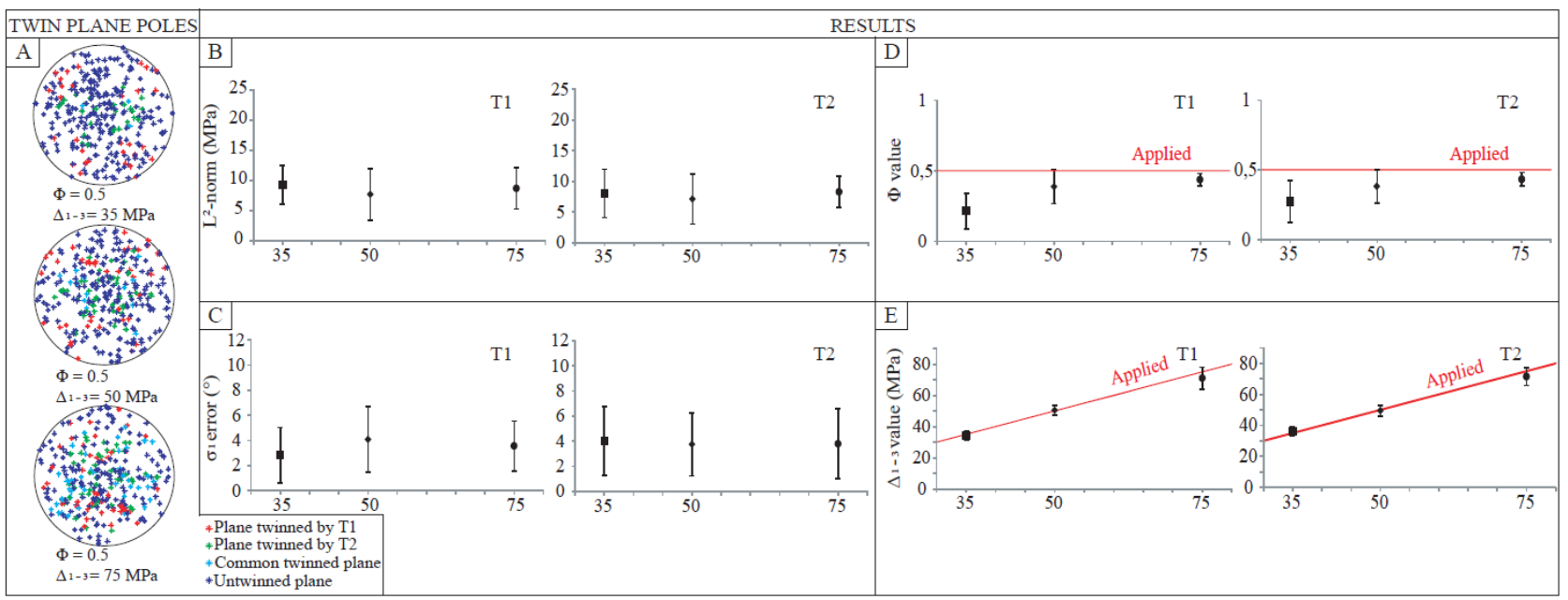

Figure 18: Inversion results of numerically-generated polyphase datasets applying B5 then B12, B1 then B13 and B6 then B14 configurations (Tables 3). A, B, C, D and E same key as in figure 14.

Additional tests were carried out to evaluate the effect of a difference of stress ratio between both applied tensors (configurations B5 then B15, B1 then B16 and B6 then B17 in table 3) (Fig. 19A). The first tensor has a N-S compression with an E-W extensional part and a stress ratio of 0. The second tensor has been rotated with respect to the first tensor by $30^{\circ}$ and corresponds to a stress ratio of 0.5 . Results show that the total error $\left(\mathrm{L}^{2}-\mathrm{norm}\right)$ is increasing with the applied differential stress and the same for the deviation (Fig. 19B). For principal stress orientation, the error is of 4-7 ${ }^{\circ}$ for a deviation of $\pm 3-4^{\circ}$ for both tensors (Fig. 19C). The stress ratio is very well reconstructed with 601 a negligible error $\left(<10^{-2}\right)$ and a negligible deviation for the first tensor and an error of $-0.1(-10 \%)$ and a deviation of less than $\pm 0.1(10 \%)$ for the second tensor (Fig. 19D). The differential stress is 603 well retrieved by the inversion process but there is a slight increase of the deviation width for both tensors and a slight error for the second tensor with an applied differential stress of $75 \mathrm{MPa}$ (Fig. 


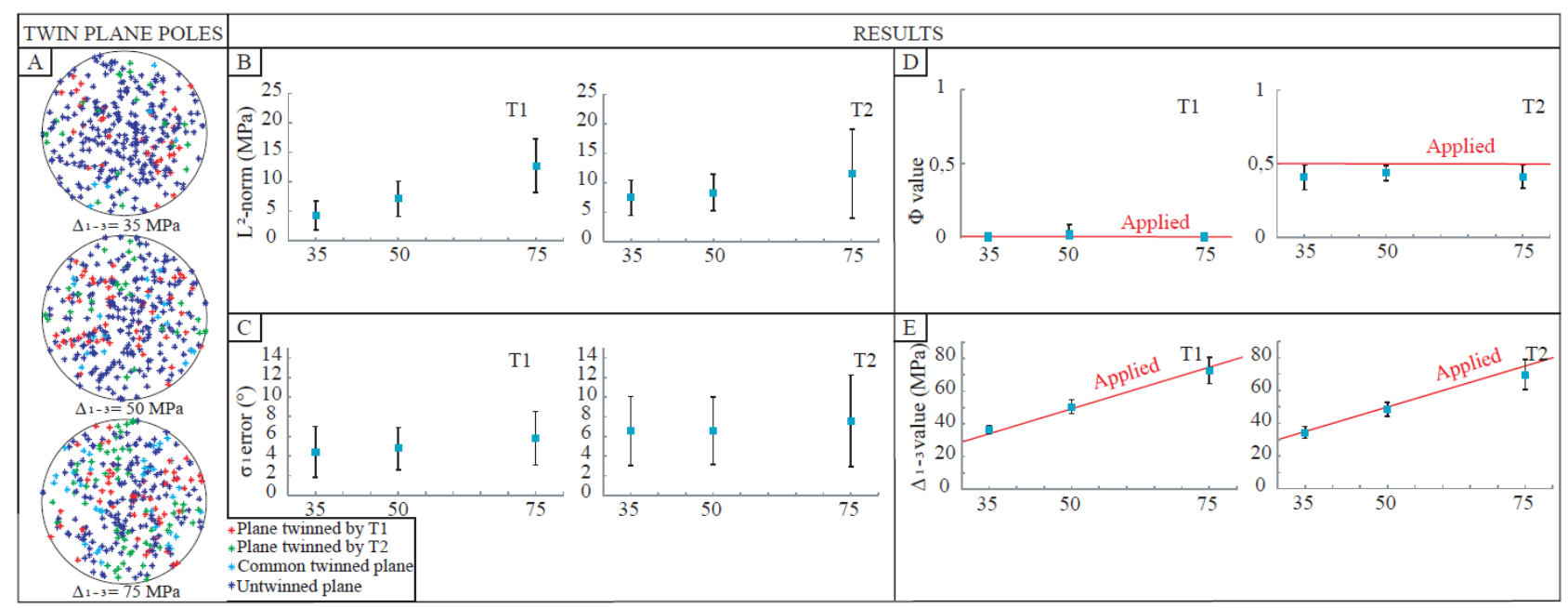

Figure 19: Inversion results of numerically-generated polyphase datasets of B5 then B15, B1 then B16 and B6 then B17 configurations (Table 3). A, B, C, D and E same key as in figure 14.

has a differential stress of $50 \mathrm{MPa}$ and the second tensor with $\mathrm{N} 030^{\circ}$ trending $\sigma 1$ has a differential stress of 35 or $75 \mathrm{MPa}$ (configurations B1 then B7 and B1 then B8 in table 3). Tensors are very close and these configurations are the most difficult in terms of separation because of the overlap of twin data explained by the two tensors. As previously, the distribution of grain sizes (i.e. CRSS value) is a 614 pseudo-Gaussian centered on a CRSS value of $10 \mathrm{MPa}$ (Fig. 20B \& B'). Figure 20B \&B' show the 615 distribution of shared twinned planes of both tensors. It can be observed that there is a higher number of shared twinned planes in figure 20B' than in figure 20B where the differential stress of the second tensor equals $75 \mathrm{MPa}$. This is explained by the fact that both tensors are very close and an increase of the differential stress value increases the number of common twinned planes (progressively 619 overlapping spherical caps in the sense of Yamaji, 2015a). Figure 20A \& A' show that the principal 620 stress orientations and stress ratio are well estimated but, as observed previously, there is an increase 621 of error on the differential stress value for the second tensor at high differential stress (Fig. 20A'). 
For the configuration shown in figure $20 \mathrm{~A}$, the error is of $4-7^{\circ}$ with a deviation of $\pm 2-3^{\circ}$ for principal stress orientations, less than $0.1(10 \%)$ for the stress ratio, $1-3 \mathrm{MPa}(2-8 \%)$ with a deviation of $\pm 2-3$ $\mathrm{MPa}(4-8 \%)$ for the differential stress. For the configuration shown in figure $20 \mathrm{~A}^{\prime}$, the error is of $6^{\circ}$ with a deviation of $\pm 2^{\circ}$ for principal stress orientations, $0.02(2 \%)$ for the stress ratio and about 2-5.5

$\mathrm{MPa}(4-7 \%)$ with a deviation of $\pm 3-5 \mathrm{MPa}(6-7 \%)$ for the differential stress.
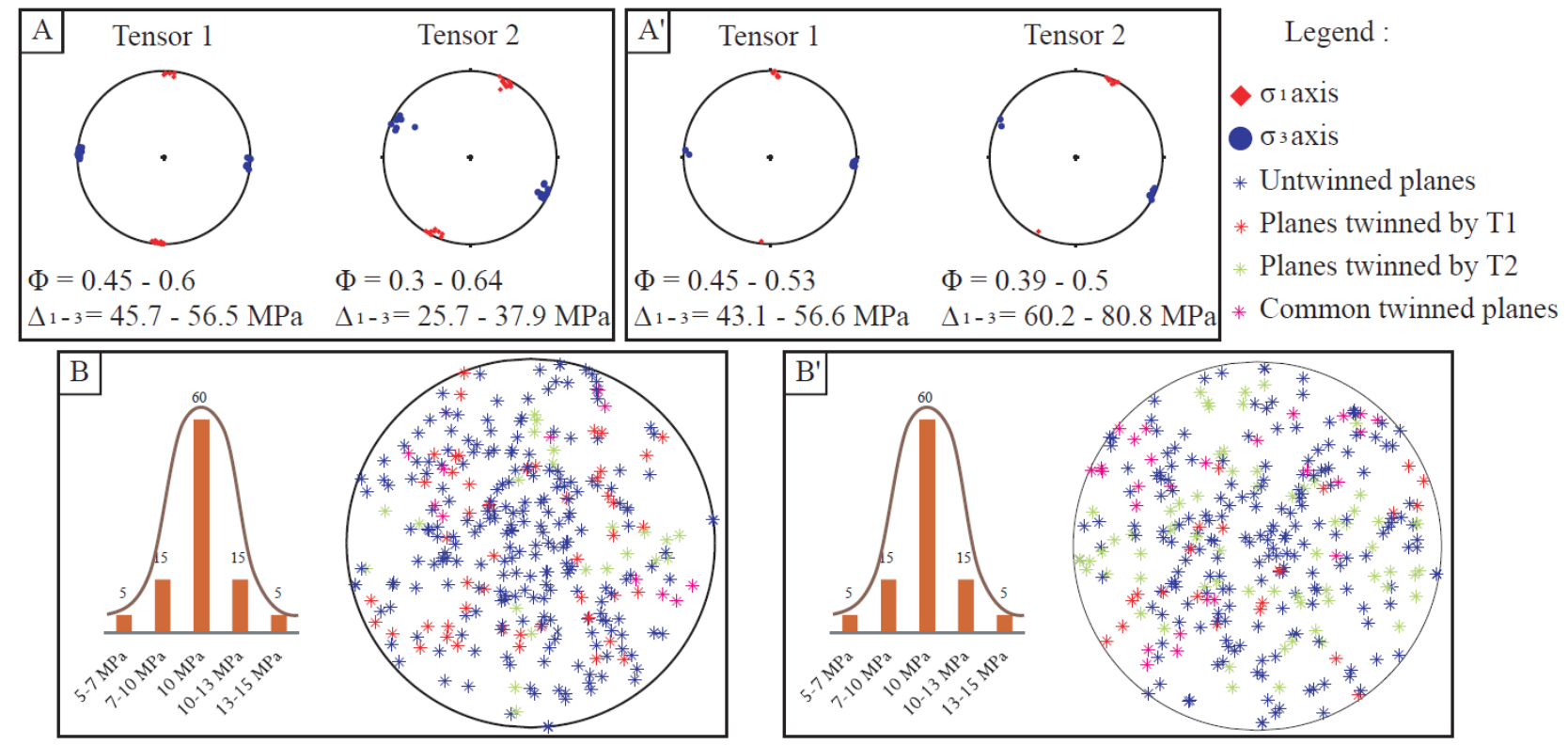

Figure 20 : Inversion results of numerically-generated polyphase datasets B1 then B7 and B1 then B8 configurations (Table 3). A and $\mathrm{A}^{\prime}$ ) Results of the inversion for each configuration. B and B') Histograms of the repartition of grain sizes and an example of stereographic projection (Wulff lower hemisphere) of twinned/untwinned plane poles.

\section{Interpretation and discussion of the results of inversion of numerically} generated datasets using CSIT-2

\subsection{Overall efficiency of CSIT-2 to determine paleostress from monophase and}

polyphase datasets

\subsubsection{Determination of the principal stress orientations}

For monophase datasets with a perfectly homogeneous grain size principal stress orientations are fully retrieved (Fig. 9). When a slight dispersion around the mean grain size is added, the average 
of about $4^{\circ}$ (by considering a constant CRSS value of $10 \mathrm{MPa}$ ) (Fig. 11). With two distinct grain size

636 classes with a pseudo-gaussian distribution, the error on the principal stress orientations is the same 637 than previously (Fig. 12). The same observations can be made for the results of dataset inversion with 638 a bias incorporation of 10 or $25 \%$ (by randomly changing twinned planes into untwinned planes) (Fig. 639 13). A slightly larger deviation in the principal stress orientations is observed with an increase of bias 640 incorporation in datasets (25\%). Thus, CSIT-2 can accurately and precisely reconstruct principal 641 stress orientations from monophase datasets in all configurations even with a high incorporation of 642 bias.

As a result, for a naturally deformed monophase sample with homogeneous grain size, the methodological uncertainty associated with the determination of principal stress orientations does not exceed $\pm 8^{\circ}$.

For polyphase datasets with one grain size class, the determination of the principal stress axes is also accurate and precise, the maximum error reaching $10^{\circ}$ on average with a maximum deviation of $\pm 5^{\circ}$ (Fig. 14-20). This higher error compared to monophase datasets is due to the fact that CSIT2 (as for CSIT) is able to estimate parameters of the stress tensor (orientations of principal stress axes and stress ratio) while the fifth parameter (non-dimensional differential stress) is found later by 651 considering a constant CRSS value of $10 \mathrm{MPa}$. In the cases of datasets with different grain sizes, applied tensors may consequently not cause twinning of some favorably oriented twin planes. These

653 planes therefore act as misclassified twinned planes (e.g., bias) during the inversion process. In fact, 654 small grains have a higher CRSS value and the applied resolved shear stress value may thus be too 655 low to cause twinning along them. This can slightly affect the determination of principal stress 656 orientation. The incorporation of bias in monophase and polyphase datasets makes it slightly more difficult to accurately and precisely determine principal stress orientations (Fig. 13 and 15). This is due to the 659 strong influence of the penalization function in the choice of the tested tensors. If the penalization 
660 function value exceeds 0.5 , the tensor is not selected (see section 3.2). Depending on the orientation 661 of untwinned planes with respect to the tensor, this value can exceed 0.5 , leading to an error on 662 principal stress orientations. The error on principal stress orientations is of $6^{\circ}$ and $10^{\circ}$ for the first and 663 the second tensors when they differ of $30^{\circ}$ in the orientation of principal stress axes with a deviation 664 of $\pm 5^{\circ}$ (for $10 \%$ bias).

Gagala (2009) stated that the CSIT was not able to separate very close tensors. In contrast, 666 CSIT-2 makes it possible to unambiguously and reliably reconstruct superimposed tensors with only 667 $30^{\circ}$ of difference between $\sigma_{1}$ axes, hence with a high percentage of shared twinned planes.

The orientations of the principal stress axes are thus the best constrained parameters, because the 3D spatial orientation of twinned planes provides strong constraints on possible orientations of stress axes. This observation was the basis of the Turner's (1953) analysis of calcite twins.

In nature it is very rare to have a sample having recorded just one phase (one applied tensor).

672 Thus, the user will be more interested by the accuracy for polyphase cases (two or more applied 673 tensors). If the tensors are very close in principal stress orientations (i.e. $30^{\circ}$ rotation between 674 principal stress axes between both tensors), the error is $10^{\circ}$ with a deviation of $\pm 5^{\circ}$ (Fig. 14-16). Thus, 675 the maximum uncertainty in calculated principal stress orientations is $\pm 15^{\circ}$. For cases with very 676 different applied tensors in term of orientations (i.e. around $90^{\circ}$ rotation between principal stress axes 677 between both tensors), the error is $4^{\circ}$ with and deviation of $\pm 2^{\circ}$, hence the maximum uncertainty is $678 \pm 6^{\circ}$ (Fig. 17). In the case of $\sigma 2-\sigma 3$ stress permutation between both applied tensors the error is $4^{\circ}$ 679 with a deviation of $\pm 4^{\circ}$, so the maximum uncertainty is therefore $\pm 8^{\circ}$ (Fig. 18).

As a result, the maximum methodological uncertainty associated with determination of 681 principal stress orientations from a naturally deformed sample with an homogeneous grain size using 
In general, the stress ratio is accurately and precisely retrieved in monophase and polyphase datasets, with bias of 10 or $25 \%$ and high differential stress (Fig. 13 and 15).

For monophase cases, the error is negligible with a deviation of \pm 0.04 , so the maximum uncertainty for monophase cases is \pm 0.04 (4\%) on the stress ratio value. For polyphase cases with $30^{\circ}$ of difference between principal stress axes of the two applied tensors, the error is of $0.03(3 \%)$ with and deviation of $\pm 0.08(8 \%)$, so the maximum uncertainty is of $\pm 0.1(10 \%)$. For tensors with $90^{\circ}$ of difference between principal stress axes the error is of $0.02(2 \%)$ with a deviation of \pm 0.05 (5\%), so the maximum uncertainty is of $\pm 0.07(7 \%)$. For all these configurations CSIT-2 is very accurate and precise for the stress ratio determination. For tensors with a permutation of $\sigma_{2}$ and $\sigma_{3}$ axes, the error is expectedly higher, $0.3(30 \%)$ for low differential stress and decrease until $0.07(7 \%)$ for high differential stress with a deviation of $\pm 0.1(10 \%)$. This last configuration is the most difficult one because at low applied differential stress the number of shared twinned planes is very high and the program will tend to find one tensor with an extreme value of stress ratio. Extreme stress ratio values (in practice $\Phi=0$ ) make it possible to take into account more twinned planes for two tensors with similar $\sigma_{1}$ axes. With increasing differential stress, the percentage of shared twinned planes between tensors decreases and allows to more accurately and precisely find the stress ratio values.

As a result, the maximum methodological uncertainty associated with the determination of the stress ratio from a naturally deformed sample with an homogeneous grain size using CSIT-2 can be considered $\pm 0.4(40 \%)$; the usual uncertainty is expected to be about $\pm 0.1(10 \%)$.

\subsubsection{Determination of differential stress magnitudes}

In monophase datasets with one grain size class and no bias (Fig. 9-10), differential stresses are retrieved with a deviation that does not exceed $3 \mathrm{MPa}$. The loss of information on the determination of differential stress, when the applied stress is greater than $50 \mathrm{MPa}$ as highlighted by Yamaji (2015a), is not observed (maximum error of $2 \mathrm{MPa}$ for an applied differential of $75 \mathrm{MPa}$ ). However, this effect is clearly observed with monophase datasets with two different grain size classes 
(see §5.3) or with polyphase datasets (Fig. 11-12).

It is noticeable that, among the 5 parameters of the deviatoric stress tensor, the differential stress is clearly the less constrained parameter. This is mainly due to the sensitivity of the CRSS to grain size and to the difficulty to take this effect into account. The error may reach $20 \%$ in polyphase cases at high applied differential stresses. Results from monophase datasets show a good estimate of the differential stress compared to polyphase datasets. For differential stress lower than $50 \mathrm{MPa}$ the error does not exceed $2 \mathrm{MPa}$ for monophase datasets without bias. The results of the analysis of monophase datasets with two grain size classes point toward the need for dividing the dataset into subsets of homogeneous grain sizes before inversion in order to better estimate the differential stresses.

In polyphase cases with one grain size class and incorporation of $10 \%$ of bias, the maximum misfits on the differential stress value is observed at high applied differential stress (75 MPa). This occurs in all cases except for the configuration with a rotation of $90^{\circ}$ (Fig. 18) of the principal stress axes. This configuration gives the most accurate and precise values for all parameters of the deviatoric stress tensors (regarding polyphase cases). For cases with two applied tensors with a difference of principal stress orientation of $30^{\circ}$, the error is of $1-6 \mathrm{MPa}(12 \%)$ for applied differential stress equal or below $50 \mathrm{MPa}$ and of 3-15 MPa (20\%) above $50 \mathrm{MPa}$ (Fig. 16). The deviation can reach \pm 2-9 $\mathrm{MPa}(18 \%)$ in the worst cases for differential stress applied equal or below $50 \mathrm{MPa}$ and $\pm 10-13 \mathrm{MPa}$ (17\%) above $50 \mathrm{MPa}$ applied.

As a result, the maximum methodological uncertainty associated with determination of differential stresses from a naturally deformed sample with a homogeneous grain size using CSIT-2 can be considered $\pm 37 \%$; this rather high value is expected for high differential stresses. The usual uncertainty is expected to be about $\pm 25-30 \%$.

\subsection{Influence of heterogeneous grain size}

Several configurations of grain sizes have been tested in order to determine the robustness of CSIT-2 to varying grain sizes. As observed when dealing with monophase datasets, a high variability 
of grain sizes does not affect the accuracy of the determination of principal stress orientations or stress ratio. However, and especially in the case of two grain size classes (Fig. 11-12), there is a loss of information for differential stresses. This loss is higher at high differential stress. This is due to the application of a constant CRSS value of $10 \mathrm{MPa}$ for all grains. As mentioned previously, several authors concurred to say that the CRSS value is strongly gain size dependent (Newman, 1994; Rowe and Rutter, 1990). Thus, the CRSS value has to be adapted for each grain size. The main problem is our poor knowledge regarding the evolution of the CRSS value with grain size.

Principal stress orientations are generally accurately and precisely determined by CSIT-2, although there are few cases at low applied differential stress showing bad results (mainly on the dip value of principal stress axis). This is due to the fact that the software calculates the 4 parameters using $\left(\sigma_{1}-\sigma_{3}\right)=1$ without taking into account the different grain sizes. Thus, at low differential stress, twinning in small grains (with high CRSS values) cannot be activated due to the differential stress of the tensor even if they are favorably oriented regarding the stress tensor orientation. In these cases, having two different grain sizes can bias the results.

\subsection{Influence of optical measurement bias}

The influence of the incorporation of bias in the datasets appears to be complex. It depends on the orientation of misclassified planes regarding to the applied tensor. If a randomly misclassified plane has a low applied resolved shear stress value, then the effect of the misclassification on the results is insignificant. If the randomly misclassified plane has a high applied resolved shear stress value regarding the applied tensor, then there is a strong influence on the penalization function, which consequently increases. Because the selection of tested tensors relies partly on the penalization function value, if this value is too high (above the limit fixed by the user), the tensor will be discarded, even if this tolerance toward a value of 0.5 allows to be less restrictive than the previous method (CSIT). The CSIT-2 selection process may miss "good" tensors depending on the orientation of the misclassified untwinned planes. It is to date impossible to predict how it affects the orientations of 
principal stress axes and other parameters because it is depending on the resolved shear stress applied on this incompatible untwinned planes and how many there are. Few tests tend to show that the principal stress orientations can be strongly affected, especially the dip of the stress axes, the azimuths being well retrieved. The stress ratio and the differential stress are not affected by the inaccuracy of the principal stress orientations. They remain well retrieved.

Our results show that the CSIT-2 method is overall robust for twin datasets with up to $25 \%$ of incorporated bias. Considering the breaks in slope on the curves of the different parameters as a function of the increasing number of twinned planes explained (Fig. 3 and 13F) leads to accurate results even at such high bias (see sections 3.2 and 4 for more details), but at the cost of an increase of the penalization function (up to 1.5) and also of the incorporation of incompatible untwinned planes (number of incompatible untwinned planes over number of compatible twinned planes + number of incompatible untwinned planes, Fig. 13E, up to 1.5). However, for natural cases the amount of optical bias is not a priori known and an untwinned plane seen as incompatible for the inversion is not necessary an optical measurement bias. It is therefore recommended, when analyzing (blindly) a naturally deformed sample, to consider with care any solution tensor that would incorporate (more than) $30 \%$ of incompatible untwinned planes and a penalization function value that would exceed 1.5 (Fig. 13E). The geological setting helps determine whether the solution-tensor is significant or not.

\subsection{Practical way to optimize data inversion}

The optical bias, likely due to errors in measurements using a U-stage can have a significant effect (on the orientation of principal stress axes) despite the improvement of the technique. To circumvent this difficulty, an index of uncertainty in the untwinned status of a twin plane has been established for measurements of natural samples. This way, the user can double-check the status and, in case of doubt, even change the status of the plane of poor confidence in the dataset. Note however that this will be applied only on untwinned planes which appeared at low percentage of twinned planes to be explained and therefore on which the tensor exerts a high resolved shear stress value. 
In order to minimize the optical bias and measurement errors and to get information from

785 completely untwinned grains, twin data are currently being measured using EBSD rather than using

786 a U-stage (Parlangeau et al., 2015). Optical bias consequently falls down to $0 \%$. However, natural heterogeneities at the grain scale (stress shadows, heterogeneous grain-to-grain stress transmission) can of course not be circumvented and their weight on the quality of results can only be estimated by comparing natural (non-perfect) data and numerical data. The decrease of incorporated bias has a positive effect on the accuracy of the stress results (see comparison of monophase results without/with bias in section 5.3).

Overall, the least constrained parameter of the reconstructed deviatoric stress tensors is the differential stress magnitude, especially when applied differential stresses are greater than $75 \mathrm{MPa}$. Combining inversion of calcite twin data with fracture analysis and rock experiments (e.g., Lacombe, 2007) may however help test the validity and the meaning of determined stress magnitudes against the mechanics of rock masses. Our study finally highlights that sorting the whole dataset into subclasses of homogeneous grain size before inversion is the best way to reliably define the 5 stress tensor parameters, especially differential stresses. That is the reason why it is strongly recommended to estimate the grain size distribution in a natural twin datasets and to apply separately the inversion technique to homogeneous grain size classes, provided the number of available twin is sufficient to 801 secure a reliable stress tensor computation. In that case, provided each class yields similar accurate 802 principal stress orientations and stress ratio, assigning a different value of the critical resolved shear stress to each class as a function of the mean grain size should provide more reliable (and expectedly consistent) estimates of differential stresses (see section 7). Taking into account the grain-size dependence of the CRSS in the inversion process is therefore a strong requirement; further work 806 should focus on providing better constraints on the variation of the CRSS as a function of grain size 807 (work in progress). 
Few studies have focused on estimating uncertainties on the results of the former CSIT (Lacombe

810 and Laurent, 1996, 1992; Laurent et al., 2000). In order to illustrate the improvement on stress

811 determination brought by using CSIT-2 instead of CSIT, we provide hereafter a comparison of the

812 stress results obtained from both techniques when applied to polyphase datasets since Gạgała (2009)

813 has casts some doubt on the ability of CSIT to well retrieve close superimposed stress tensors. The

814 first configuration tested is the one applying a N-S compression with a stress ratio of 0.5 and a

815 differential stress of $50 \mathrm{MPa}$, the second applied tensor is turner of $30^{\circ}$ between $\sigma_{1}$ axes with a stress

816 ratio of 0.5 and a differential stress of $50 \mathrm{MPa}$ (configurations $\mathrm{B} 25$ then $\mathrm{B} 27$ in table 3). The second

817 configuration is exactly the same than the first one but with a differential stress of $75 \mathrm{MPa}$ for both

818 tensors (configurations B26 then B28 in table 3). The last tested configuration is applying a first

819 tensor with a N-S strike-slip regime $\left(\sigma_{1}: \mathrm{N} 180-0\right.$ and $\left.\sigma_{3}: \mathrm{N} 270-0\right)$ with a stress ratio of 0.5 and a

820 differential stress of $35 \mathrm{MPa}$. The second tensor applied is also a N-S strike-slip regime but with a

821 permutation of the $\sigma_{2}$ and $\sigma_{3}$ axes $\left(\sigma_{1}: \mathrm{N} 180-0\right.$ and $\left.\sigma_{3}: \mathrm{N} 270-90\right)$ with a stress ratio of 0.5 and a

822 differential stress of $35 \mathrm{MPa}$ (configurations B24 then B29 in table 3).

\begin{tabular}{|c|c|c|c|c|c|c|c|c|}
\hline \multirow{2}{*}{. } & \multicolumn{8}{|c|}{ First tensor } \\
\hline & \multicolumn{2}{|r|}{ Applied } & \multicolumn{2}{|r|}{ CSIT } & \multicolumn{2}{|c|}{ Applied } & \multicolumn{2}{|r|}{ CSIT-2 } \\
\hline \multirow{3}{*}{$\begin{array}{l}\text { Stress axes orientation } \\
\qquad\left(^{\circ}\right)\end{array}$} & $\sigma_{1}$ & N180-0 & $\sigma_{1}$ & N185-8 & $\sigma_{1}$ & N180-0 & $\sigma_{1}$ & N1-1 \\
\hline & $\sigma_{2}$ & N90-90 & $\sigma_{2}$ & N46-79 & $\sigma_{2}$ & N90-90 & $\sigma_{2}$ & $\mathrm{~N} 84-80$ \\
\hline & $\sigma_{3}$ & N270-0 & $\sigma_{3}$ & N275-7 & $\sigma_{3}$ & N270-0 & $\sigma_{3}$ & N92-10 \\
\hline Stress ratio & & 0,5 & & 0,53 & & 0,5 & & 0,48 \\
\hline Differential stress (MPa) & & 50 & & 45,3 & & 50 & & 50 \\
\hline$L^{2}$-norm (MPa) & \multicolumn{4}{|c|}{9,6} & \multicolumn{4}{|c|}{6.2} \\
\hline
\end{tabular}

\begin{tabular}{|c|c|c|c|c|c|c|c|c|}
\hline \multirow{5}{*}{$\begin{array}{l}\text { Stress axes orientation } \\
\qquad\left(^{\circ}\right)\end{array}$} & \multicolumn{8}{|c|}{ Second tensor } \\
\hline & \multicolumn{2}{|r|}{ Applied } & \multicolumn{2}{|r|}{ CSIT } & \multicolumn{2}{|r|}{ Applied } & \multicolumn{2}{|r|}{ CSIT-2 } \\
\hline & $\sigma_{1}$ & N30-0 & $\sigma_{1}$ & N36-5 & $\sigma_{1}$ & N30-0 & $\sigma_{1}$ & N28-4 \\
\hline & $\sigma_{2}$ & N120-90 & $\sigma_{2}$ & N117-85 & $\sigma_{2}$ & N120-90 & $\sigma_{2}$ & N160-84 \\
\hline & $\sigma_{3}$ & N300-0 & $\sigma_{3}$ & N126-5 & $\sigma_{3}$ & N300-0 & $\sigma_{3}$ & N298-4 \\
\hline Stress ratio & & 0,5 & & 0,06 & & 0,5 & & 0,45 \\
\hline Differential stress (MPa) & & 50 & & 41,7 & & 50 & & 48,6 \\
\hline $\mathrm{L}^{2}$-norm (MPa) & \multicolumn{4}{|c|}{17,5} & \multicolumn{4}{|c|}{5,0} \\
\hline
\end{tabular}

Table 5: Results of the inversion using CSIT and CSIT-2 of the configuration B25 then B27. The L2norm is the distance between the applied tensor and the solution-tensor found by CSIT or CSIT-2.

823 For the first configurations B25 then B27 (Table 3), the results are shown in table 5. $\mathrm{L}^{2}$-norm 
824 value for solution-tensors found by CSIT-2 are way lower than the one calculated using CSIT. 825 Especially for the configuration B27 (Table 3) where CSIT finds a stress ratio of 0.06 instead of 0.5 826 which significantly increases the $\mathrm{L}^{2}$-norm value. The stress axis orientations are retrieved by both 827 CSIT and CSIT-2. The differential stress is also well retrieved by both techniques with less error on 828 CSIT-2 results than using CSIT.

829 For the second configurations B26 then B28 (Table 3), the results are shown in the table 6 . This 830 configuration is different from the first one only about the differential stress value which is higher, $83175 \mathrm{MPa}$ for both tensors. As said previously, there is a loss of the information about differential stress 832 between 50-75 MPa of applied differential stresses. Again, the distance between the applied tensors 833 and the solution tensors is lower for the results using CSIT-2 than for CSIT. Especially for the first 834 tensor (B26 in table 3) for which CSIT strongly overestimates the differential stress. The tensor found 835 is clearly intermediate between the first and the second applied tensors.

\begin{tabular}{|c|c|c|c|c|c|c|c|c|}
\hline \multirow{5}{*}{$\begin{array}{l}\text { Stress axes orientation } \\
\qquad\left(\left(^{\circ}\right)\right.\end{array}$} & \multicolumn{8}{|c|}{ First tensor } \\
\hline & \multicolumn{2}{|r|}{ Applied } & \multicolumn{2}{|r|}{ CSIT } & \multicolumn{2}{|c|}{ Applied } & \multicolumn{2}{|r|}{ CSIT-2 } \\
\hline & $\sigma_{1}$ & N180-0 & $\sigma_{1}$ & N196-3 & $\sigma_{1}$ & N180-0 & $\sigma_{1}$ & N7-4 \\
\hline & $\sigma_{2}$ & N90-90 & $\sigma_{2}$ & N78-84 & $\sigma_{2}$ & N90-90 & $\sigma_{2}$ & N198-86 \\
\hline & $\sigma_{3}$ & N270-0 & $\sigma_{3}$ & N286-6 & $\sigma_{3}$ & N270-0 & $\sigma_{3}$ & N97-1 \\
\hline Stress ratio & & 0,5 & & 0,45 & & 0,5 & & 0,46 \\
\hline Differential stress (MPa) & & 75 & & 92,4 & & 75 & & 75,9 \\
\hline $\mathrm{L}^{2}$-norm (MPa) & \multicolumn{4}{|c|}{35,6} & \multicolumn{4}{|c|}{13,8} \\
\hline
\end{tabular}

\begin{tabular}{|c|c|c|c|c|c|c|c|c|}
\hline \multirow{5}{*}{$\begin{array}{l}\text { Stress axes orientation } \\
\qquad\left({ }^{\circ}\right)\end{array}$} & \multicolumn{8}{|c|}{ Second tensor } \\
\hline & \multicolumn{2}{|r|}{ Applied } & \multicolumn{2}{|r|}{ CSIT } & \multicolumn{2}{|c|}{ Applied } & \multicolumn{2}{|r|}{ CSIT-2 } \\
\hline & $\sigma_{1}$ & N30-0 & $\sigma_{1}$ & N25-9 & $\sigma_{1}$ & N30-0 & $\sigma_{1}$ & N25-5 \\
\hline & $\sigma_{2}$ & N120-90 & $\sigma_{2}$ & N116-81 & $\sigma_{2}$ & N120-90 & $\sigma_{2}$ & N121-85 \\
\hline & $\sigma_{3}$ & N300-0 & $\sigma_{3}$ & N295-9 & $\sigma_{3}$ & N300-0 & $\sigma_{3}$ & N115-5 \\
\hline Stress ratio & & 0,5 & & 0,49 & & 0,5 & & 0,49 \\
\hline Differential stress (MPa) & & 75 & & 74,7 & & 75 & & 65,3 \\
\hline $\mathrm{L}^{2}$-norm (MPa) & \multicolumn{4}{|c|}{12,3} & \multicolumn{4}{|c|}{11,9} \\
\hline
\end{tabular}

Table 6: Results of the inversion using CSIT and CSIT-2 of the configuration B26 then B28. The L²norm is the distance between the applied tensor and the solution-tensor found by CSIT or CSIT- 2 .

For the last configurations B24 then B29 (Table 3), the results are shown in table 7. This configuration is the most difficult to retrieve for CSIT-2 as shown in figure 18, especially for the 838 stress ratio value. In table 7, the $\mathrm{L}^{2}$-norm distance calculated between applied and solution tensors 
839 shows that, again, CSIT-2 is more accurate than CSIT. CSIT finds a tensor which allows the

840 permutation between $\sigma_{3}$ and $\sigma_{2}$ axes with a stress ratio very low of 0.03 .

\begin{tabular}{|c|c|c|c|c|c|c|c|c|}
\hline \multirow{5}{*}{$\begin{array}{l}\text { Stress axes orientation } \\
\qquad\left({ }^{\circ}\right)\end{array}$} & \multicolumn{8}{|c|}{ First tensor } \\
\hline & \multicolumn{2}{|r|}{ Applied } & \multicolumn{2}{|r|}{ CSIT } & \multicolumn{2}{|c|}{ Applied } & \multicolumn{2}{|r|}{ CSIT-2 } \\
\hline & $\sigma_{1}$ & N180-0 & $\sigma_{1}$ & N1-15 & $\sigma_{1}$ & N180-0 & $\sigma_{1}$ & $\mathrm{~N} 1-0$ \\
\hline & $\sigma_{2}$ & N90-90 & $\sigma_{2}$ & N204-73 & $\sigma_{2}$ & N90-90 & $\sigma_{2}$ & N266-86 \\
\hline & $\sigma_{3}$ & N270-0 & $\sigma_{3}$ & N93-6 & $\sigma_{3}$ & N270-0 & $\sigma_{3}$ & N91-4 \\
\hline Stress ratio & & 0,5 & & 0,59 & & 0,5 & & 0,49 \\
\hline Differential stress (MPa) & & 35 & & 32,5 & & 35 & & 33,5 \\
\hline $\mathrm{L}^{2}$-norm (MPa) & \multicolumn{4}{|c|}{7,1} & \multicolumn{4}{|c|}{2,2} \\
\hline
\end{tabular}

\begin{tabular}{|c|c|c|c|c|c|c|c|c|}
\hline \multirow{5}{*}{$\begin{array}{l}\text { Stress axes orientation } \\
\left.\text { ( }{ }^{\circ}\right)\end{array}$} & \multicolumn{8}{|c|}{ Second tensor } \\
\hline & \multicolumn{2}{|r|}{ Applied } & \multicolumn{2}{|r|}{ CSIT } & \multicolumn{2}{|r|}{ Applied } & \multicolumn{2}{|r|}{ CSIT-2 } \\
\hline & $\sigma_{1}$ & N180-0 & $\sigma_{1}$ & N179-4 & $\sigma_{1}$ & N180-0 & $\sigma_{1}$ & N184-0 \\
\hline & $\sigma_{2}$ & N90-0 & $\sigma_{2}$ & N88-19 & $\sigma_{2}$ & N90-0 & $\sigma_{2}$ & N94-0 \\
\hline & $\sigma_{3}$ & N270-90 & $\sigma_{3}$ & N281-71 & $\sigma_{3}$ & N270-90 & $\sigma_{3}$ & N1-90 \\
\hline Stress ratio & & 0,5 & & 0,03 & & 0,5 & & 0,45 \\
\hline Differential stress ( $\mathrm{MPa}$ ) & & 35 & & 37,3 & & 35 & & 32,3 \\
\hline $\mathrm{L}^{2}$-norm $(\mathrm{MPa})$ & \multicolumn{4}{|c|}{15,0} & \multicolumn{4}{|c|}{2,9} \\
\hline
\end{tabular}

Table 7: Results of the inversion using CSIT and CSIT-2 of the configuration B24 then B29. The L2norm is the distance between the applied tensor and the solution-tensor found by CSIT or CSIT- 2 .

As a result, although some stress configurations are similarly retrieved by CSIT and CSIT-2.

CSIT-2 being always more accurate than CSIT. Some other more complex stress configurations are using CSIT-2 will expectedly improve the reliably of stress determination in polyphase settings in forthcoming tectonic studies.

\section{Application to naturally deformed polyphase samples}

In order to test the CSIT-2 and its ability to derive geologically meaningful paleostresses, the technique is applied on samples collected from limestones of Tithonian-Aptian age (Maiolica formation) in the Monte Nero anticline located within the Umbria-Marches tectonic domain of the northern Apennines (Italy). The Monte Nero-Serra Santa is an arcuate fold with a backlimb dipping $30^{\circ}$ towards W-SW and an intensively folded forelimb, where the strata are locally overturned (Fig.21). Microstructural investigations revealed occurrence of several sets of joints and veins (mode 

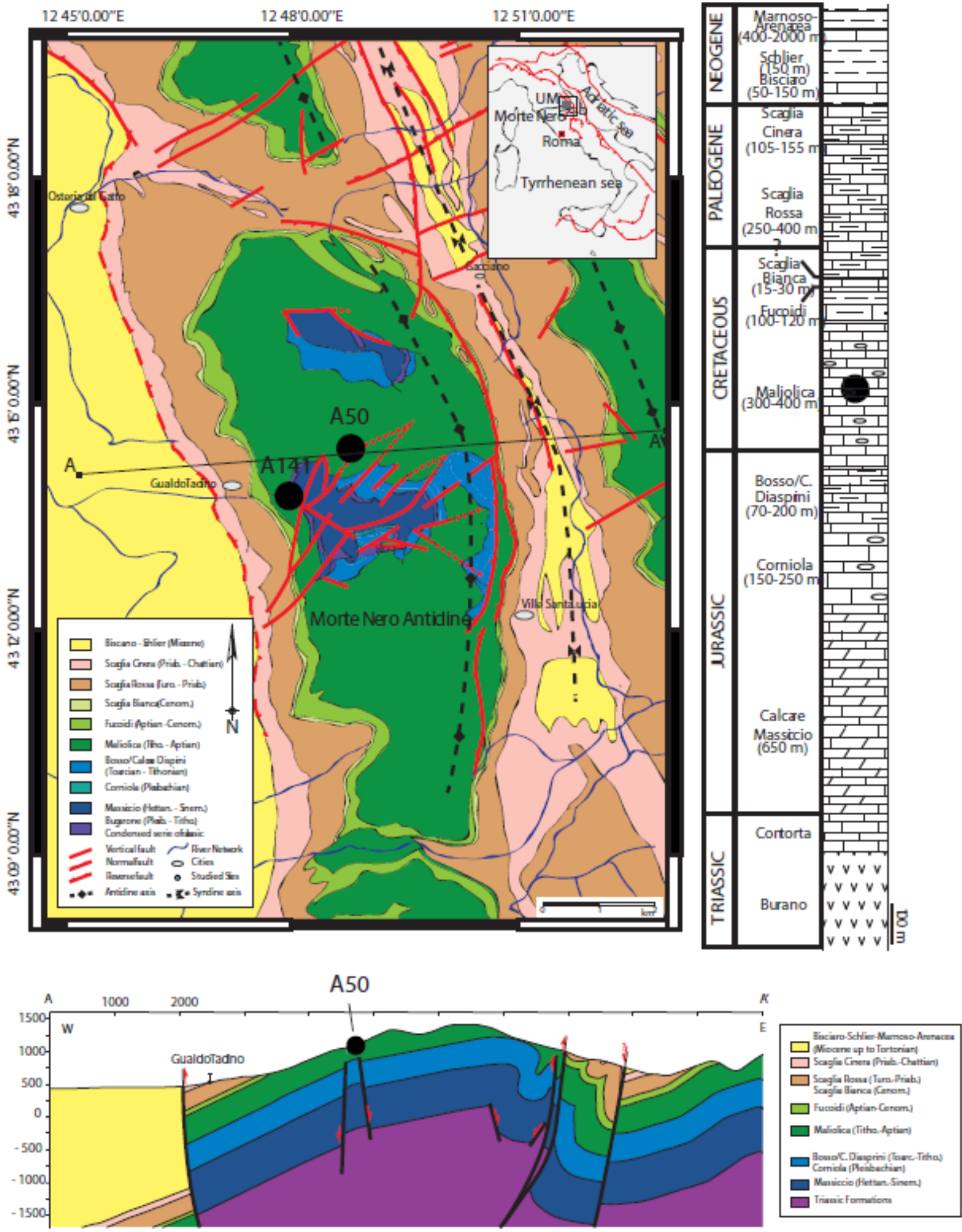

Figure 21: Simplified geological map and stratigraphic log of the Monte Nero anticline (northern Appenines) with location of the sample studied (modified after Beaudoin et al., 2016).

In the western limb of the fold, we selected a first sample containing two intersecting veins 


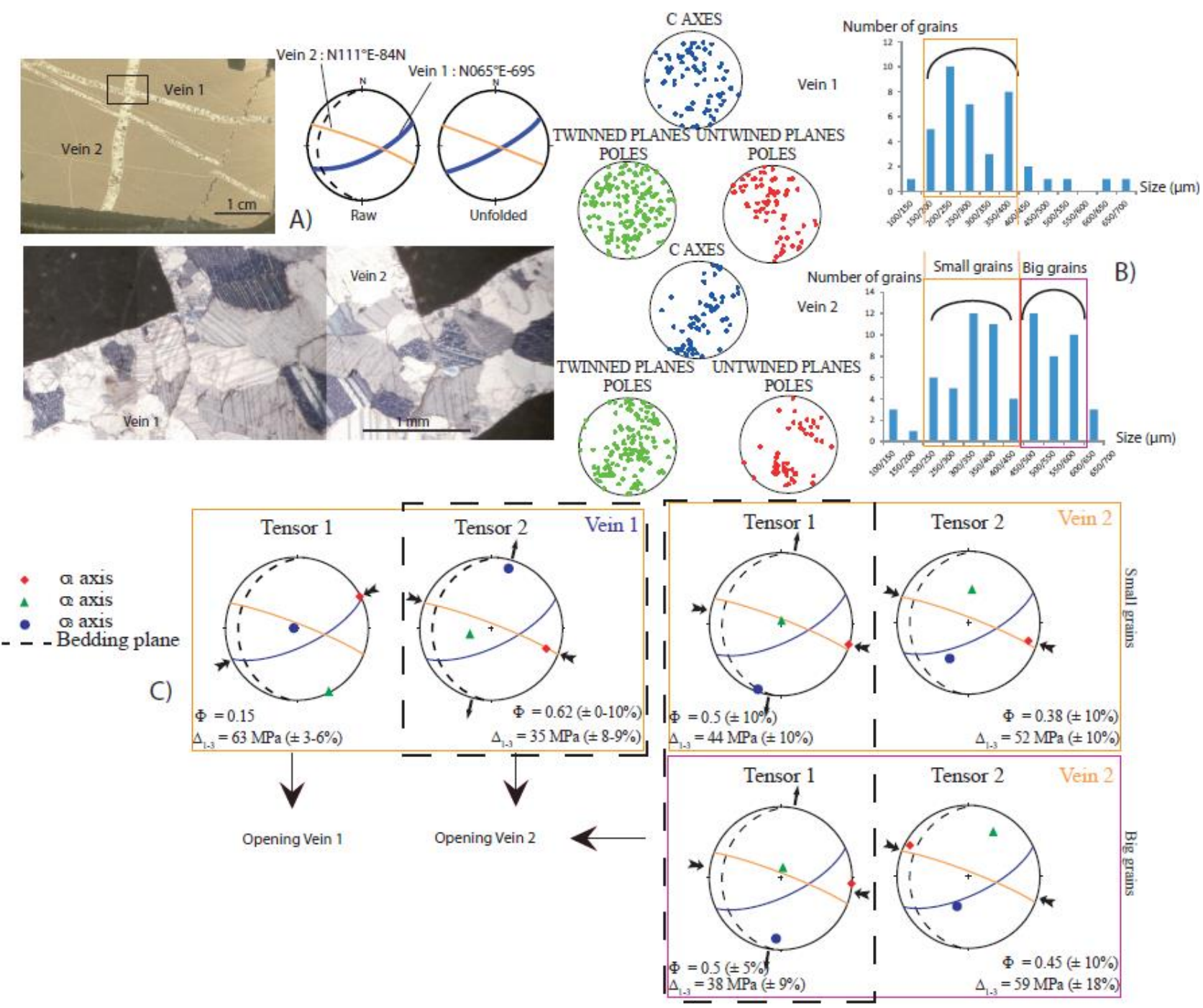

Figure 22: Inversion results of naturally deformed polyphase datasets. A) Picture of a thin section (crossed polars) showing crosscutting veins, together with stereographic projection of measured veins (Wulff's lower hemisphere equal area projection). B) Plots of $\mathrm{C}$ axes, and poles of twinned and untwinned planes from veins 1 and 2 together with distribution of related grain sizes. C) Results of inversion of calcite twins from vein 1 and vein 2 (for more details, see text). For the vein 1, the first tensor is oriented with $\sigma_{1}:$ N63-2 and $\sigma_{3}: 297-87$ and the second tensor is oriented with $\sigma_{1}:$ N11021 and $\sigma_{3}: 15-13$. For the vein 2 small grains, the first tensor is oriented with $\sigma_{1}:$ N105-1 and $\sigma_{3}$ : 195-3 and the second tensor is oriented with $\sigma_{1}:$ N108-16 and $\sigma_{3}: 215-47$. For the vein 2 big grains, the first tensor is oriented with $\sigma_{1}:$ N95-1 and $1227 \sigma_{3}: 185-20$ and the second tensor is oriented with $\sigma_{1}: \mathrm{N} 300-8$ and $\sigma_{3}: 200-53$.

This chronology between the two vein sets is documented in many places at the scale of the fold and 
861 is therefore statistically valid at the regional scale. These vein sets are associated with sets of bed862 perpendicular stylolites of tectonic origin and are parallel to the teeth they exhibit (peaks oriented 863 N050 and N090-110). This observation supports the fact that both fracture sets developed under sub864 horizontal compression ( $\sigma_{1}$ axis) before folding.

We carried out U-stage measurements of calcite twins within these two veins from 3 mutually 866 perpendicular thin-sections. Calcite grains display thin $(0.5 \mu \mathrm{m})$ and rectilinear e-twins, which cross 867 the crystals over all their length (Fig. 22A). These characteristics indicate that calcite grains probably 868 underwent weak strain, $3-4 \%$ at most, under conditions of temperature lower than $150^{\circ} \mathrm{C}-200^{\circ} \mathrm{C}$ 869 (Ferrill et al., 2004). For each vein, we measure and report the orientations of C axes and of twinned 870 and untwinned planes and we estimate grain sizes using the 3 thin-sections (Fig. 22B) to avoid as 871 much as possible bias related to 2D observations. We could define a single grain size class for Vein 8721 and two grain sizes for Vein 2. One should keep in mind that because these classes must contain a 873 sufficient number of grains for the inversion to be statistically valid (at least 30 grains), they must not 874 be too narrow. Inversion is carried out for each grain size class independently. For Vein 1 the CRSS 875 considered is of $9 \mathrm{MPa}$. For Vein 2, the CRSS value is of $10 \mathrm{MPa}$ for the lower grain size (200-400 $876 \mu \mathrm{m})$ and $7.5 \mathrm{MPa}$ for the higher grain size $(400-600 \mu \mathrm{m})$. Note that although the grain size class of 877 Vein 1 is similar to the smaller grain size class of Vein 2, the adopted CRSS value are slightly different 878 (9 and $10 \mathrm{MPa}$, respectively). This is because the CRSS is also dependent on internal twinning strain, 879 which is roughly estimated using twin density to be higher in Vein 2 than in Vein 1 . This way to take 880 into account strain hardening is similar to Rocher et al. (2004) and Amrouch et al (2010).

Taking into account the crosscutting relationships between the veins (Fig. 22A), twinned 882 calcite from Vein 1 expectedly recorded (at least) the state of stress responsible for its opening, but 883 also the later state of stress responsible for the formation of Vein 2. In contrast, calcite from Vein 2 884 should not have recorded the state of stress responsible for the earlier opening of Vein 1 but should 885 have expectedly recorded the state of stress responsible for its own formation. 
887 process within each vein. Vein 1 records a N050 $\pm 6^{\circ}$ compression with a stress ratio of 0.15 , a differential stress of $63 \mathrm{MPa} \pm 3-6 \%$ and a N110 $\pm 6^{\circ}$ compression associated with perpendicular extension, a stress ratio of $0.62 \pm 0.1$, a differential stress of $35 \mathrm{MPa} \pm 8 \%$. Vein 2 yields a stress tensor similar to the second tensor from vein 1 . The uncertainties associated with the previous results are estimated from the interpretation of the analyses on synthetic datasets. Results from the inversion process are consistent across grain size ranges.

A relative chronology between successive twinning stresses can be established through consideration of orientation of computed stress axes (as well as of stress ratio) with respect to vein orientation. The simple underlying idea is that a stress tensor determined from the calcite grains filling a vein with a $\sigma_{3}$ axis perpendicular to the vein strike is likely related to the vein opening while other tensors with stress axes inconsistent with the vein geometry likely reflect later, post-opening stress regimes (Lacombe, 2010).

The compressional configuration of tensor 1 from Vein 1 with subhorizontal $\sigma_{1}$ axis lying parallel to the vein and subvertical $\sigma_{3}$ axis, is not at first glance consistent with opening of Vein 1. 901 But the low value of the stress ratio supports that $\sigma_{2}$ and $\sigma_{3}$ are very close in magnitudes hence prone 902 to switch, so the computed $\sigma_{3}$ axis is consistent with vein opening in a strike-slip / compressional 903 stress regime. This tensor should be considered as predating tensor 2 , which is therefore likely post904 opening. The strike-slip configuration of tensor 2 from Vein 1 with subhorizontal $\sigma_{1}$ and $\sigma_{3}$ axes lying 905 within and perpendicular to Vein 2, respectively, is in perfect agreement with opening of this vein. 906 This means that Vein 1 recorded the stress regime responsible for opening of Vein 2 which postdates 907 Vein 1. Similarly, the strike-slip configuration of tensor 1 from Vein 2 with subhorizontal $\sigma_{1}$ and $\sigma_{3}$ 908 axes lying within and perpendicular to the vein, respectively, is in perfect agreement with opening of 909 Vein 2, and should be considered as predating tensor 2 from Vein 2. Tensor 2 from Vein 2 is therefore 910 a likely post-opening stress tensor, which reflects a later change from strike-slip to compressional 
stress configuration, the $\sigma_{1}$ axis remaining unchanged.

Interestingly, tensor 2 from Vein 1 and tensor 1 from Vein 2 are also very close in terms of stress magnitudes with only 0.1 of difference in stress ratio and $9 \mathrm{MPa}$ maximum difference in differential stress magnitude $\left(\sigma_{1}-\sigma_{3}\right)$.

On a methodological point of view, these results show that despite the natural heterogeneity of the calcite filling the veins, CSIT-2 captures the stress regimes consistent with opening of successive regional vein sets which developed during successive substages of the tectonic evolution of the Monte Nero anticline (Beaudoin et al., 2016). The pre-folding N050 compression is related to the regional Apenninic compression which formed the Umbria-Marches domain and started during Tortonian times (Barchi et al., 2012; Billi et al., 2007; Marshak et al., 1982; Storti and Salvini, 2001; Tavani et al., 2008). The subsequent pre-folding N090 to N110 compression is interpreted as a perturbation of the Apenninic compression caused by a N-S striking fault at depth beneath the fold, likely inherited from Tethyan rifting, and which reactivation as a high-angle thrust controlled the structure of the Monte Nero anticline (Beaudoin et al., 2016.).

Finally, differential stresses obtained from vein $1(63 \mathrm{MPa} \pm 3-6 \%$ for the first tensor and 35 $\mathrm{MPa} \pm 8-9 \%)$ and $2(38-44 \mathrm{MPa} \pm 9-10 \%)$ are in good agreement with independent differential stress estimates based on tectonic stylolite roughness paleopiezometry at the same locations (Beaudoin et al., 2016). Taking into account an average burial of the Maiolica formation of $2700 \mathrm{~m}$ at the time of deformation as derived from the stratigraphic succession, these values are also in good agreement with predicted ranges of differential stress values for compressional strike-slip stress regime (opening of Vein 1) and strike-slip stress regime (opening of Vein 2) at this depth based on differential stress/depth relationship in the crust as reported by Lacombe (2007).

As shown in figure 23A, C axes and poles to twinned and untwinned planes for both veins show a non homogeneous spatial distribution. Even though the high level of consistency of the results argues in favor of the robustness of the technique to such potential bias, we also analyzed another sample containing a vein from the same set (N050 trending bed-perpendicular set) than V1 from the 
937 former sample that shows a more homogeneous distribution of $\mathrm{C}$ axes and poles to twinned and 938 untwinned planes. This sample is located (Fig. 23A) in the western part of the Monte Nero anticline, 939 also in the Massicio formation.
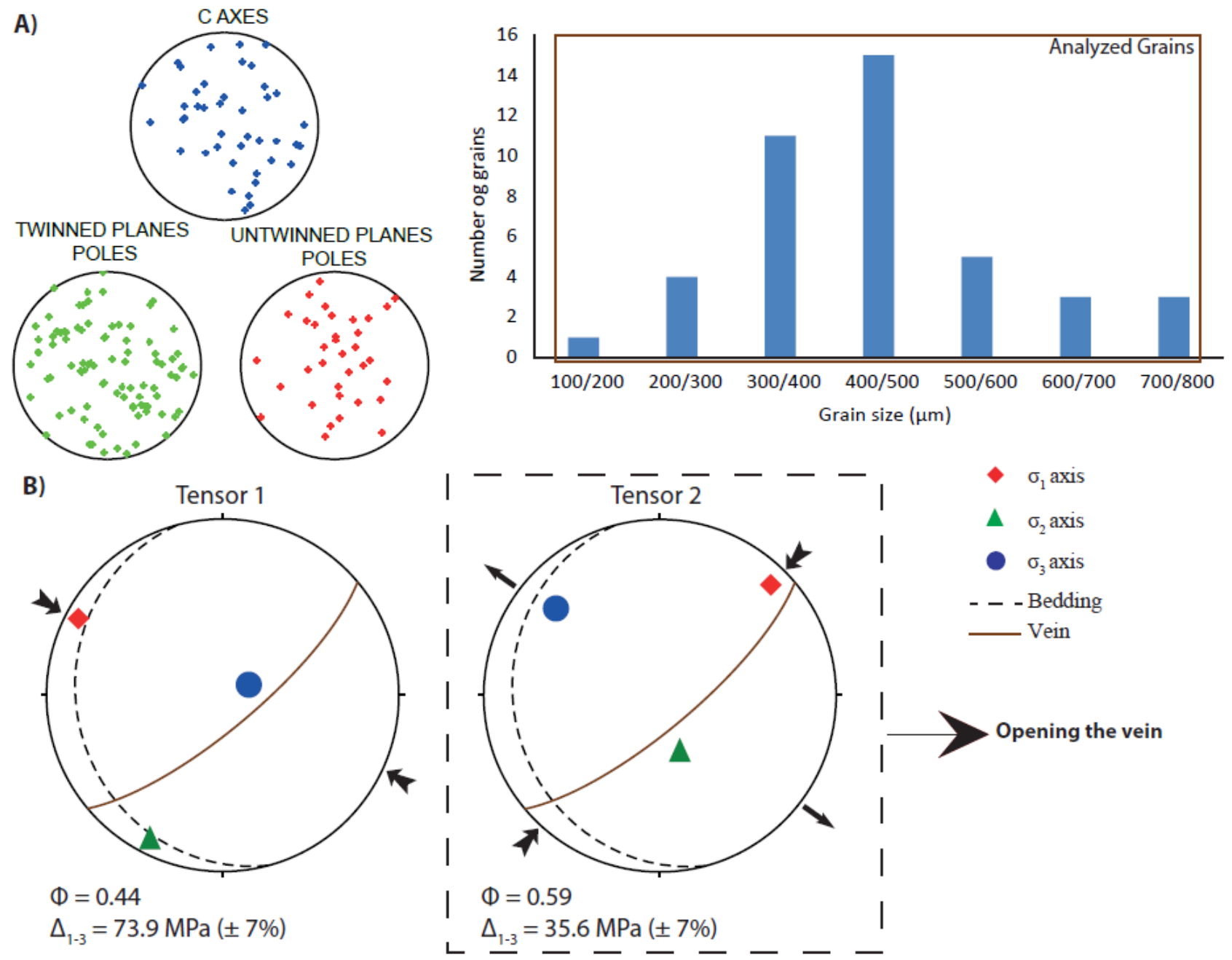

Figure 23: Inversion results of naturally deformed polyphase datasets. A) Plots of C axes, and poles of twinned and untwinned planes with distribution of related grain sizes. B) Results of inversion of calcite twins (for more details, see text). The first tensor is oriented with $\sigma_{1}: \mathrm{N} 298-8$ and $\sigma_{3}: 68-77$. The second tensor is oriented with $\sigma_{1}: \mathrm{N} 45-12$ and $\sigma_{3}: 310-24$.

The results of the inversion show a strike-slip configuration with a N45 tending $\sigma_{1}$ axis and a 941 perpendicular horizontal $\sigma_{3}$ axis (Fig. 23B) consistent with the opening of the vein (N50-75S in 942 present attitude). The maximum differential stress is $35.6 \mathrm{MPa}( \pm 7 \%)$ with a stress ratio of 0.59 . 943 Another tensor has been revealed by the inversion that corresponds to a N118 compression with a 944 differential stress value of $73.9 \mathrm{MPa}( \pm 7 \%)$ and a stress ratio of $0.44 \mathrm{The} 118^{\circ}$ compression likely 945 postdates the strike-slip regime with a N45 $\sigma_{1}$ axis which is consistent with vein opening. 
In terms of orientations, both stress tensors are consistent with those determined

947 independently from the nearby former sample, which supports the ability of the technique to provide 948 consistent, regionally significant stress tensors. The consistency of the results from both samples 949 further indicates that a slight preferred orientations of the $\mathrm{C}$ axes and poles to twinned and untwinned planes exerts a negligible bias on stress tensor determination using CSIT-2. associated with a much greater uncertainty than those derived from the tests on synthetic datasets because the low number of grains precluded any separation of the natural dataset into classes of homogeneous grain size, so that the $9 \mathrm{MPa}$ value of the CRSS adopted for calculation is badly constrained. In spite of this additional effect, the values for the second sample remain of the same order than those derived from the first sample and fall within the range of differential stresses reported by Beaudoin et al. (2016) in the Monte Nero anticline.

\section{Conclusions}

Inversion of calcite twin data is to date an efficient approach to determine both paleostress orientations and magnitudes in the upper crust. This paper proposes a new technique inspired from the CSIT (Etchecopar, 1984) but which circumvents its main limitations. The ability of the new technique to detect, to separate and to determine stress tensors from monophase and polyphase twin datasets including measurements errors or various grain sizes is demonstrated by numerous tests on synthetic (numerically generated) twin datasets. In contrast to Gągała (2009), Rez and Melichar (2010) and Yamaji (2015), who do not report any application of their techniques to natural data, this paper shows that apart from synthetic samples for which the physical problems related to stress transmission,

969 grain rheological properties (e.g., elasticity) and grain-scale stress heterogeneities are neglected, the 970 technique also yields reliable paleostresses from naturally deformed polyphase samples. It is however 971 strongly advised to handle the results provided by any stress inversion techniques with care, and to 
972 have a critical look at the results, making the best use of available geological information (e.g., 973 relative chronology) to guide the inversion procedure and separation of superimposed stress tensors. 974 The main technical limitations of paleostress reconstructions from calcite twins to date, 975 whatever the inversion technique is used, are related to (1) potential errors in optical measurements; 976 (2) the influence of grain size and grain size distribution in the deformed aggregates, and (3) our 977 insufficient knowledge of the variations of the CRSS value for twinning with grain size. While the 978 first is likely to be overcome by twin data acquisition using EBSD (Parlangeau et al., 2015) and the 979 second is possibly circumvented by defining several grain size classes and treating them separately, 980 understanding the influence of the grain size distribution and characterizing the way the CRSS varies 981 with changing grain size are challenges for forthcoming studies.

Acknowledgements:

984 B. Célérier, K. Amrouch, A. Yamaji and the editor J.-P. Avouac are thanked for their constructive 985 comments that greatly improved the manuscript. We also thank a lot E. Kholer for his help in drafting 986 this manuscript. Authors are most obliged to N. Beaudoin who provides the natural samples from the 987 Monte Nero anticline. 
Amrouch, K., Beaudoin, N., Lacombe, O., Bellahsen, N., Daniel, J.-M., 2011. Paleostress magnitudes in folded sedimentary rocks. Geophys. Res. Lett. 38, n/a-n/a. doi:10.1029/2011GL048649

Amrouch, K., Lacombe, O., Bellahsen, N., Daniel, J.-M., Callot, J.-P., 2010. Stress and strain patterns, kinematics and deformation mechanisms in a basement-cored anticline: Sheep Mountain Anticline, Wyoming. Tectonics 29, n/a-n/a. doi:10.1029/2009TC002525

Arboit, F., Amrouch, K., Collins, A.S., King, R., Morley, C., 2015. Determination of the tectonic evolution from fractures faults, and calcite twins on the southwestern margin of the Indochina Block. Tect 34, 1576-1599. doi:10.1002/2015TC003876

Arboit, F., Amrouch, K., Morley, C., Collins, A.S., King, R., n.d. Paleostress magnitudes in the Khao Khwang fold-thrust belt, new insights into the tectonic evolution of the indonesian orogeny in central Thailand. Tectonophysics.

Barchi, M.R., Alvarez, W., Shimabukuro, D.H., 2012. The Umbria-Marche Apennines as a double orogen: Observations and hypotheses. Ital. J. Geosci. 131, 258-271. doi:10.3301/IJG.2012.17

Beaudoin, N., Koehn, D., Lacombe, O., Lecouty, A., Billi, A., Aharonov, E., Parlangeau, C., 2016. Fingerprinting stress : stylolite and calcite twinning paleopiezometry reveal the complexity of progressive stress patterns during folding - the case of the Monte Nero anticline in the Apennines, Italy. Tectonics 35, 1687-1712. doi:10.1002/2016TC004128

Beaudoin, N., Lacombe, O., Bellahsen, N., Amrouch, K., Daniel, J.-M., 2013. Evolution of porefluid pressure during folding and basin contraction in overpressured reservoirs: Insights from the Madison-Phosphoria carbonate formations in the Bighorn Basin (Wyoming, USA). Mar. Pet. Geol. doi:10.1016/j.marpetgeo.2013.12.009

Billi, A., Valle, A., Brilli, M., Faccenna, C., Funiciello, R., 2007. Fracture-controlled fluid circulation and dissolutional weathering in sinkhole-prone carbonate rocks from central Italy. J. Struct. Geol. 29, 385-395. doi:10.1016/j.jsg.2006.09.008

Burkhard, M., 1993. Calcite twins, their geometry, appearance and significance as stress-strain markers and indicators of tectonic regime: a review. J. Struct. Geol. 15, 351-368. doi:10.1016/0191-8141(93)90132-T

De Bresser, J.H.P., Spiers, C.J., 1997. Strength characteristics of the r, f, and c slip systems in calcite. Tectonophysics 272, 1-23. doi:10.1016/S0040-1951(96)00273-9

Etchecopar, A., 1984. Etudes des états de contrainte en tectonique cassante et simulations de déformations plastiques (approche mathématiques). Université des Sciences et Techniques du Languedoc.

Etchecopar, A., Vasseur, G., Daignieres, M., 1981. An inverse problem in microtectonics for the determination of stress tensors from fault striation analysis. J. Struct. Geol. 3, 51-65. doi:10.1016/0191-8141(81)90056-0

Ferrill, D.A., 1998. Critical re-evaluation of differential stress estimates from calcite twins in coarse-grained limestone. Tectonophysics 285, 77-86. doi:10.1016/S0040-1951(97)00190-X

Ferrill, D.A., Morris, A.P., Evans, M.A., Burkhard, M., Groshong, R.H., Onasch, C.M., 2004. Calcite twin morphology: a low-temperature deformation geothermometer. J. Struct. Geol. 26, 1521-1529. doi:10.1016/j.jsg.2003.11.028

Gạgała, Ł., 2009. Reliability of selected procedures of stress inversion and data separation for inhomogeneous populations of calcite twins and striated faults: Insights from numerical experiments. Int. J. Earth Sci. 98, 461-479. doi:10.1007/s00531-007-0262-3 
1033

1034

1035

1036

1037

1038

1039

1040

1041

1042

1043

1044

1045

1046

1047

1048

1049

1050

1051

1052

1053

1054

1055

1056

1057

1058

1059

1060

1061

1062

1063

1064

1065

1066

1067

1068

1069

1070

1071

1072

1073

1074

1075

1076

1077

1078

Gągała, Ł., 2009. Performance maps - a tool for examination of reliability of procedures for automatic separation of heterogeneous fault / slip and calcite twin data. ORSGET 296, $292-$ 296.

Jamison, W.R., Spang, J.H., 1976. Use of calcite twin lamellae to infer differential stress. Geol. Soc. Am. Bull. 87, 868. doi:10.1130/0016-7606(1976)87<868:UOCTLT>2.0.CO;2

Kulikowski, D., Amrouch, K., 2017. Combining geophysical data and calcite twin stress inversion to refine the tectonic history of subsurface and offshore provinces: A case study on the CooperEromanga basin, Australia. Tectonics 36, 515-541.

Laborde, O., 1989. Formes quadratiques et méthodes géométriques en géologie. Université des Sciences et Techniques du Languedoc.

Lacombe, O., 2010. Calcite Twins, a Tool for Tectonic Studies in Thrust Belts and Stable Orogenic Forelands. Oil Gas Sci. Technol. - Rev. d'IFP Energies Nouv. 65, 809-838. doi:10.2516/ogst/2009088

Lacombe, O., 2007. Comparison of paleostress magnitudes from calcite twins with contemporary stress magnitudes and frictional sliding criteria in the continental crust: Mechanical implications. J. Struct. Geol. 29, 86-99. doi:10.1016/j.jsg.2006.08.009

Lacombe, O., 2001. Paleostress magnitudes associated with development of mountain belts: Insights from tectonic analyses of calcite twins in the Taiwan Foothills. Tectonics 20, 834-849. doi:10.1029/2001TC900019

Lacombe, O., Angelier, J., Laurent, P., Bergerat, F., Tourneret, C., 1990. Joint analyses of calcite twins and fault slips as a key for deciphering polyphase tectonics : Burgundy as a case study. Tectonics 182, 279-300. doi:10.1016/0040-1951(90)90168-8

Lacombe, O., Laurent, P., 1996. Determination of deviatoric stress tensors based on inversion of calcite twin data from experimentally deformed monophase samples: preliminary results. Tectonophysics 255, 189-202. doi:10.1016/0040-1951(95)00136-0

Lacombe, O., Laurent, P., 1992. Determination of principal stress magnitudes using calcite twins and rock mechanics data. Tectonophysics 202, 83-93. doi:10.1016/0040-1951(92)90456-G

Lacombe, O., Malandain, J., Vilasi, N., Amrouch, K., Roure, F., 2009. From paleostresses to paleoburial in fold-thrust belts: Preliminary results from calcite twin analysis in the Outer Albanides. Tectonophysics 475, 128-141. doi:10.1016/j.tecto.2008.10.023

Laurent, P., Bernard, P., Vasseur, G., Etchecopar, A., 1981. Stress tensor determination from the study of e twins in calcite: A linear programming method. Tectonophysics 78, 651-660. doi:10.1016/0040-1951(81)90034-2

Laurent, P., Kern, H., Lacombe, O., 2000. Determination of deviatoric stress tensors based on inversion of calcite twin data from experimentally deformed monophase samples. Part II. Axial and triaxial stress experiments. Tectonophysics 327, 131-148. doi:10.1016/S00401951(00)00165-7

Laurent, P., Tourneret, C., Laborde, O., 1990. Determining deviatoric stress tensors from calcite twins: Applications to monophased synthetic and natural polycrystals. Tectonics 9, 379-389. doi:10.1029/TC009i003p00379

Marshak, S., Geiser, P.A., Alvarez, W., Engelder, T., 1982. Mesoscopic fault array of the northern Umbrian Appennine fold belt, Italy: geometry of conjugate shear by pressure-solution slip. Geol. Soc. Am. Bull. doi:10.1130/0016-7606(1982)93<1013:MFAOTN>2.0.CO;2

Nemcok, M., Kovác, D., Lisle, R.J., 1999. A stress inversion procedure for polyphase calcite twin and fault/slip data sets. J. Struct. Geol. 21, 597-611. doi:10.1016/S0191-8141(99)00053-X

Newman, J., 1994. The influence of grain size and grain size distribution on methods for estimating 
1079

1080

1081

1082

1083

1084

1085

1086

1087

1088

1089

1090

1091

1092

1093

1094

1095

1096

1097

1098

1099

1100

1101

1102

1103

1104

1105

1106

1107

1108

1109

1110

1111

1112

1113

1114

1115

1116

1117

1118

1119

1120

1121

1122

1123

1124

paleostresses from twinning in carbonates. J. Struct. Geol. 16, 1589-1601. doi:10.1016/01918141(94)90129-5

Parlangeau, C., Lacombe, O., Brisset, F., Kohler, E., Daniel, J.-M., Schueller, S., 2015. Inversion of calcite twin data for paleostress (2): EBSD as a tool for data measurements, in: European Geosciences Union.

Peeters, T.H.J.,, Rodrigues, P.., Vilanova, A., ter Haar Romeny, B.., 2009. Analysis of Distance/Similarity Measures for diffusion tensor imaging, Visualization and Processing of Tensor Fields.

Pfiffner, O.A., Burkhard, M., 1987. Determination of paleo-stress axes orientations from fault, twin and earthquake data. Ann. Tectonicae 1, 48-57.

Rez, J., Melichar, R., 2010. Peek inside the black box of calcite twinning paleostress analysis. ORSGET 30, 163-168.

Rocher, M., Cushing, M., Lemeille, F., Lozac'h, Y., Angelier, J., 2004. Intraplate paleostresses reconstructed with calcite twinning and faulting: improved method and application to the eastern Paris Basin (Lorraine, France). Tectonophysics 387, 1-21. doi:10.1016/j.tecto.2004.03.002

Rocher, M., Lacombe, O., Angelier, J., 1996. Chanical twin sets in calcite as markers of recent collisional events in a fold-and-thrust belt : Evidence from the reefal limestones of southwestern Taïwan. Tectonics 15, 984-996. doi:10.1029/96TC00625

Rocher, M., Lacombe, O., Angelier, J., Deffontaines, B., Verdier, F., 2000. Cenozoic folding and faulting in the south Aquitaine Basin ( France ): Insights from combined structural and paleostress analyses. J. Struct. Geol. 22, 627-645. doi:10.1016/S0191-8141(99)00181-9

Rosenbrock, H.H., 1960. An Automatic Method for Finding the Greatest or Least Value of a Function. Comput. J. 3, 175-184. doi:10.1093/comjnl/3.3.175

Rowe, K.J., Rutter, E.H., 1990. Palaeostress estimation using calcite twinning: experimental calibration and application to nature. J. Struct. Geol. 12, 1-17. doi:10.1016/01918141(90)90044-Y

Storti, F., Salvini, F., 2001. The evolution of a model trap in the central apennines, italy : fracture patterns, fault reactivation and development of cataclastic rocks in carbonates at the narni anticline. J. Struct. Geol. 24, 171-190.

Tavani, S., Storti, F., Salvini, F., Toscano, C., 2008. Stratigraphic versus structural control on the deformation pattern associated with the evolution of the Mt . Catria anticline, Italy 30, 664681. doi:10.1016/j.jsg.2008.01.011

Tourneret, C., 1990. Maclage et état de contraintes dans les roches carbonatées du domaine fragile. Application à des plates-formes d'avant-pays de chaînes (Pyrénées, Alpes). Université des Sciences et Techniques du Languedoc.

Tullis, T.E., 1980. The use of mechanical twinning in minerals as a measure of shear stress magnitudes. J. Geophys. Res. 85, 6263. doi:10.1029/JB085iB11p06263

Turner, F.J., 1953. Nature and dynamic interpretation of deformation lamellae in calcite of three marbles. Am. J. Sci. 251, 276-298. doi:10.2475/ajs.251.4.276

Turner, F.J., Griggs, D.T., Heard, H., 1954. Experimental deformation of calcicte crystals. Geol. Soc. Am. Bull. 65, 883. doi:10.1130/0016-7606(1954)65[883:EDOCC]2.0.CO;2

Yamaji, A., 2015a. How tightly does calcite e-twin constrain stress? J. Struct. Geol. 72, 83-95. doi:10.1016/j.jsg.2015.01.008

Yamaji, A., 2015b. Generalized Hough transform for the stress inversion of calcite twin data. J. Struct. Geol. doi:10.1016/j.jsg.2015.08.001 
1125 Yamaji, a., Sato, K., 2006. Distances for the solutions of stress tensor inversion in relation to misfit 1126 angles that accompany the solutions. Geophys. J. Int. 167, 933-942. doi:10.1111/j.13651127 246X.2006.03188.x

1128 